\title{
Dendritic Spine Dynamics Regulate the Long-Term Stability of Synaptic Plasticity
}

\author{
Cian 0'Donnell, ${ }^{1,2}$ Matthew F. Nolan, ${ }^{3}$ and Mark C. W. van Rossum ${ }^{1}$ \\ ${ }^{1}$ Institute for Adaptive and Neural Computation, and ${ }^{2}$ Neuroinformatics Doctoral Training Centre, School of Informatics, University of Edinburgh, \\ Edinburgh EH8 9AB, United Kingdom, and ${ }^{3}$ Centre for Integrative Physiology, University of Edinburgh, Edinburgh EH8 9XD, United Kingdom
}

Long-term synaptic plasticity requires postsynaptic influx of $\mathrm{Ca}^{2+}$ and is accompanied by changes in dendritic spine size. Unless $\mathrm{Ca}^{2+}$ influx mechanisms and spine volume scale proportionally, changes in spine size will modify spine $\mathrm{Ca}^{2+}$ concentrations during subsequent synaptic activation. We show that the relationship between $\mathrm{Ca}^{2+}$ influx and spine volume is a fundamental determinant of synaptic stability. If $\mathrm{Ca}^{2+}$ influx is undercompensated for increases in spine size, then strong synapses are stabilized and synaptic strength distributions have a single peak. In contrast, overcompensation of $\mathrm{Ca}^{2+}$ influx leads to binary, persistent synaptic strengths with double-peaked distributions. Biophysical simulations predict that CA1 pyramidal neuron spines are undercompensating. This unifies experimental findings that weak synapses are more plastic than strong synapses, that synaptic strengths are unimodally distributed, and that potentiation saturates for a given stimulus strength. We conclude that structural plasticity provides a simple, local, and general mechanism that allows dendritic spines to foster both rapid memory formation and persistent memory storage.

\section{Introduction}

Long-term synaptic plasticity is believed to underlie learning in the brain (Milner et al., 1998; Morris et al., 2003). Synaptic plasticity in central neurons is initiated by changes in dendritic spine $\mathrm{Ca}^{2+}$ concentration driven by presynaptic and postsynaptic neuronal activity. The $\mathrm{Ca}^{2+}$ signals are detected by molecular machinery within the spine, triggering a biochemical cascade that leads to potentiation or depression of synaptic efficacy (see Fig. 1Ai) (Zucker, 1999; Malenka and Bear, 2004). Successful memory storage requires that changes in synaptic strength persist over time. To achieve this, the synaptic plasticity machinery must remain insensitive to spontaneous $\mathrm{Ca}^{2+}$ fluctuations from ongoing neural activity. Understanding how dendritic spines and their synapses solve this trade-off between plasticity and stability is a fundamental problem for the neuroscience of memory.

Here we propose that the coupling of synaptic plasticity to structural changes in spines provides a solution to this problem. A close link between spine structural plasticity and synaptic plasticity is suggested by several experimental observations: (1) dendritic spine size is tightly correlated with the strength of the synapse it hosts (Matsuzaki et al., 2001); (2) spine size is actively regulated during synaptic plasticity (Matsuzaki et al., 2004); (3)

Received May 20, 2011; accepted Aug. 22, 2011.

Author contributions: C.O., M.F.N., and M.C.W.v.R. designed research; C.O. and M.C.W.v.R. performed research; C.O. and M.C.W.v.R. contributed unpublished reagents/analytic tools; C.O. analyzed data; C.O., M.F.N., and M.C.W.v.R. wrote the paper.

This work was supported by the Medical Research Council (C.O. and M.F.N.), the Engineering and Physical Sciences Research Council (C.O. and M.C.W.v.R.), the Biotechnology and Biological Sciences Research Council (M.F.N.), the Human Frontier Science Program (M.C.W.v.R.), and a Marie Curie Excellence grant (M.F.N.). We thank Matthias Hennig, Thomas Oertner, Clare Puddifoot, and Arianna Rinaldi for comments on the manuscript.

Correspondence should be addressed to Cian O'Donnell, Room 2.51, School of Informatics, University of Edinburgh, 10 Crichton Street, Edinburgh EH8 9AB, UK. E-mail: cian.odonnell@ed.ac.uk.

DOI:10.1523/JNEUROSCI.2520-11.2011

Copyright $\odot 2011$ the authors $\quad 0270-6474 / 11 / 3116142-15 \$ 15.00 / 0$ because of their differences in volume, small spines exhibit greater $\left[\mathrm{Ca}^{2+}\right]$ changes during synaptic activation than large spines (Nimchinsky et al., 2004; Noguchi et al., 2005; Sobczyk et al., 2005); and (4) large spines are more persistent in vivo than small spines (Grutzendler et al., 2002; Trachtenberg et al., 2002; Holtmaat et al., 2005; Zuo et al., 2005; Knott et al., 2006).

Clues to the function of the relationship between spine morphology and synaptic plasticity come from computational models that predict that spine shape and size regulate $\mathrm{Ca}^{2+}$ dynamics (Gold and Bear, 1994) and the threshold for synaptic plasticity (Kalantzis and Shouval, 2009). Nevertheless, the function and general consequences of the correlation between spine size and synaptic strength remain poorly understood. In particular, it is not known how dynamic structural plasticity of dendritic spines affects either the ability of synapses to encode new information or the robustness of long-term synaptic information storage in the face of ongoing neural activity.

To address these issues, we investigate the consequences of dendritic spine structural plasticity for synaptic $\mathrm{Ca}^{2+}$ signaling. We find that the exact form of the relationship between $\mathrm{Ca}^{2+}$ influx and spine size crucially determines long-term synaptic stability, synaptic strength distribution, and whether synapses store information as a binary or continuous variable. We then use a detailed biophysical model to predict that spine structural plasticity enhances the stability of hippocampal CA3-CA1 pyramidal neuron synapses while allowing them to store information as a continuous variable. Our results unify several disparate experimental findings and suggest a novel mechanism for rapid but robust synaptic information storage.

\section{Materials and Methods}

Simulations and analysis were carried out using MATLAB (Mathworks) (see Figs. 1-6) and MCell (Stiles et al., 1996) (see Fig. 7). The nomenclature of voltage-gated $\mathrm{Ca}^{2+}$ channels and glutamate receptors follows the 
guidelines of Catterall et al. (2005) and Collingridge et al. (2009), respectively.

$\mathrm{Ca}^{2+}$ signaling-dependent plasticity rule. Throughout the study, we model the $\mathrm{Ca}^{2+}$ dependence of synaptic plasticity as follows: low or baseline spine $\mathrm{Ca}^{2+}$ concentration cause no change in synaptic strength, $\mathrm{Ca}^{2+}$ concentrations above a moderate threshold trigger depression, and $\mathrm{Ca}^{2+}$ concentrations above a higher threshold cause potentiation (see Fig. 1 Aii). These assumptions are consistent with many previous studies (Bienenstock et al., 1982; Bear et al., 1987; Lisman, 1989; Cummings et al., 1996; Hansel et al., 1997; Yang et al., 1999; Cho et al., 2001; Cormier et al., 2001; Shouval et al., 2002; Ismailov et al., 2004). We formulate the $\mathrm{Ca}^{2+}$-dependent plasticity rule as the difference of two sigmoid functions, as follows:

$$
\Delta w=\frac{\eta_{p}}{1+e^{-\left(\left[C a^{2+}\right]-\theta_{p}\right) / \sigma_{d}}}-\frac{\eta_{d}}{1+e^{-\left(\left[C a^{2+}\right]-\theta_{d}\right) / \sigma_{d}}},
$$

where $\Delta w$ is the change in synaptic strength, $\eta$ sets the magnitude of plasticity events, $\left[\mathrm{Ca}^{2+}\right]$ is the spine $\mathrm{Ca}^{2+}$ concentration $(\mu \mathrm{M})$, and $\theta$ $(\mu \mathrm{M})$ and $\sigma(\mu \mathrm{M})$ set the offset and steepness of the sigmoids, respectively. The subscripts $p$ and $d$ denote potentiation and depression, respectively. Because we let $\theta$ and $\sigma$ be independent of synaptic strength, we are implicitly assuming that the properties of the molecular $\mathrm{Ca}^{2+}$ detectors and plasticity machinery do not vary with synaptic strength.

General conditions for exact, under-, and overcompensation. We denote the $\mathrm{Ca}^{2+}$ influx during a stimulus as $J_{\mathrm{Ca}}$. The scenario (exact/under-/ overcompensation) can be determined by examining the relationship between $J_{\mathrm{Ca}}$ and spine volume, $V_{\mathrm{sp}}$. To maintain constant stimulusevoked changes in spine $\left[\mathrm{Ca}^{2+}\right]$, an increase in spine volume, $\Delta V_{\mathrm{sp}}$, must be matched by a proportional increase in $\mathrm{Ca}^{2+}$ influx, $\Delta J_{\mathrm{Ca}}$. Exact compensation is achieved only when $\frac{\Delta J_{\mathrm{Ca}}}{J_{\mathrm{Ca}}}=\frac{\Delta V_{\mathrm{sp}}}{V_{\mathrm{sp}}}$. For example, a $50 \%$ increase in spine volume would require a corresponding $50 \%$ increase in $\mathrm{Ca}^{2+}$ influx. Undercompensation occurs if there is not a sufficient increase in $\mathrm{Ca}^{2+}$ influx to counter the fractional change in spine volume, as follows:

$$
\frac{\Delta J_{\mathrm{Ca}}}{J_{\mathrm{Ca}}}<\frac{\Delta V_{\mathrm{sp}}}{V_{\mathrm{sp}}},
$$

Analogously, overcompensation occurs when the fractional increase in $\mathrm{Ca}^{2+}$ influx is greater than the fractional change in spine volume, as follows:

$$
\frac{\Delta J_{\mathrm{Ca}}}{J_{\mathrm{Ca}}}>\frac{\Delta V_{\mathrm{sp}}}{V_{\mathrm{sp}}} .
$$

Quantitative changes in the scaling relationship between spine size and $\mathrm{Ca}^{2+}$ influx that do not cross these boundaries will change only the quantitative aspects of synaptic strength dynamics, but not their qualitative behavior.

As an example, consider a spine with a spherical head, surface area $\left(A_{s p}\right)$, and volume $V_{\mathrm{sp}}$ where spine $\mathrm{Ca}^{2+}$ influx arises solely from GluNs (NMDA receptors). If the number of spine GluNs scales proportionally to spine surface area and is set at 10 channels $/ \mu \mathrm{m}^{2}$, then:

$$
\begin{aligned}
J_{\mathrm{Ca}}\left(V_{\mathrm{sp}}\right) & =10 j_{\mathrm{Ca}} A_{\mathrm{sp}} \\
& =10 j_{\mathrm{Ca}}\left((36 \pi)^{1 / 3} V_{\mathrm{sp}}^{2 / 3}\right) \\
& \propto V_{\mathrm{sp}}^{2 / 3},
\end{aligned}
$$

where $j_{\mathrm{Ca}}$ is the $\mathrm{Ca}^{2+}$ influx through a single GluN. Then, for small spine volume changes:

$$
\frac{\Delta J_{\mathrm{Ca}}}{J_{\mathrm{Ca}}}=\frac{2}{3} \frac{\Delta V_{\mathrm{sp}}}{V_{\mathrm{sp}}} .
$$

In this case, any increase in spine volume is accompanied by a smaller increase in $\mathrm{Ca}^{2+}$ influx. Hence, scaling GluN number proportional to spine surface area leads to undercompensation. Keeping the number of GluNs constant also leads to undercompensation, as does any other con- dition where there is an insufficient increase in $\mathrm{Ca}^{2+}$ influx to match a change in spine volume.

Integrate-and-fire model. The subthreshold voltage of the leaky integrate-and-fire neuron (see Figs. 2C, 3) was modeled as

$$
\frac{d V}{d t}=\left(-V+R_{\text {in }} I_{\text {syn }}\right) / \tau_{m},
$$

where $V_{m}$ is the membrane potential $(\mathrm{mV}), R_{\text {in }}$ is the input resistance (1 $\mathrm{G} \Omega), I_{\mathrm{syn}}=\sum_{n=1}^{N_{\mathrm{sy}}} i_{\mathrm{syn}}$ is the summed synaptic input current (pA), and $\tau_{m}$ is the membrane time constant $(10 \mathrm{~ms})$. When the voltage reaches threshold, $V_{\mathrm{th}}=20 \mathrm{mV}$, a spike is fired and the voltage is reset to zero. We use current-based synapses where single synaptic input currents were modeled as $i_{\text {syn }}=w\left(e^{-t / \tau_{\text {decay }}}-e^{-t / \tau_{\text {rise }}}\right)$, where $w$ is the synaptic weight $(\mathrm{pA})$ and $\tau_{\text {rise }}=0.18 \mathrm{~ms}$ and $\tau_{\text {decay }}=1.8 \mathrm{~ms}$ set the waveform time course.

In this simplified model, synaptic $\mathrm{Ca}^{2+}$ signals arise solely from GluNs (NMDA receptors) (Sabatini et al., 2002; Shouval et al., 2002). The $\mathrm{Ca}^{2+}$ influx (mol/s) through GluNs is modeled as $J_{\mathrm{NMDA}}=\rho \nu$. This is the instantaneous product of a dimensionless variable representing the fraction of glutamate-bound GluNs, $\rho$, which is synapse specific, with a dimensionless voltage variable representing postsynaptic membrane potential change from a back-propagated action potential, $\nu$, which is identical for all synapses (Shouval et al., 2002). This additional voltage variable is necessary because the standard integrate-and-fire model does not describe the voltage during an action potential, which is crucial for relieving the $\mathrm{Mg}^{+}$block of GluNs. Both $\rho$ and $\nu$ were constrained between 0 and 1 , and evolved as

$\frac{d x}{d t}=-x / t$, where $x$ is either $\rho$ or $\nu\left(\tau_{\rho}=50 \mathrm{~ms}\right.$ and $\left.\tau_{\nu}=5 \mathrm{~ms}\right)$. When the synapse is activated, $\rho_{t+\Delta t}=\rho_{t}+\left(1-\rho_{t}\right) / 2$, ensuring eventual GluN saturation upon repeated activation. When a postsynaptic spike occurs, $\nu \rightarrow$ 1. The amount of spine $\mathrm{Ca}^{2+}, \mathrm{Ca}_{\mathrm{sp}}(\mathrm{mol})$, from the $\mathrm{Ca}^{2+}$ influx through GluNs follows:

$$
\frac{d C a_{\mathrm{sp}}}{d t}=J_{\mathrm{NMDA}}-C a_{\mathrm{sp}} / \tau_{\mathrm{Ca}}
$$

$\left(\tau_{\mathrm{Ca}}=20 \mathrm{~ms}\right)$. Importantly, the spine volume, $V_{\mathrm{sp}}$, is always proportional to the synaptic weight such that $V_{\mathrm{sp}}=w \times 0.01 \mu \mathrm{m}^{3} / \mathrm{pA}$ (Matsuzaki et al., 2001).

To model each of the three scenarios of exact, under-, and overcompensation, we set the magnitude of $\mathrm{Ca}^{2+}$ influx through GluNs, $J_{\mathrm{NMDA}}$ $(\mathrm{mol} / \mathrm{s})$, to be a function of spine volume, as follows:

$$
J_{\mathrm{NMDA}}\left(V_{\mathrm{sp}}\right)=k V_{\mathrm{sp}}^{\alpha},
$$

where $V_{\mathrm{sp}}$ is the spine volume $\left(\mu \mathrm{m}^{3}\right)$, and the exponent $\alpha$ determines the scenario, as explained below. $k$ is a constant $\left(k_{\text {comp }}=833.3, k_{\text {under }}=6\right.$, and $\left.k_{\text {over }}=100,000 \mathrm{~mol} / \mathrm{s} / \mu \mathrm{m}^{3 \alpha}\right)$ that is found by tuning so that spine $\mathrm{Ca}^{2+}$ transients were in the range of $0-10 \mu \mathrm{M}$. This rescaling factor, $k$, is necessary to implement the phenomenological relationship we impose between spine size and $\mathrm{GluN} \mathrm{Ca}^{2+}$ influx. The spine $\mathrm{Ca}^{2+}$ concentration following synaptic activation is related to the absolute amount of $\mathrm{Ca}^{2+}$ by the following:

$$
\left[\mathrm{Ca}^{2+}\right]=\frac{C a_{\mathrm{sp}}}{V_{\mathrm{sp}}} \propto \frac{k V_{\mathrm{sp}}^{\alpha}}{V_{\mathrm{sp}}}=k V_{\mathrm{sp}}^{\alpha-1} .
$$

If $\alpha=1$, then spine [ $\mathrm{Ca}^{2+}$ ] is independent of spine volume. This corresponds to the compensating scenario. In contrast, if $\alpha<1$, then spine $\left[\mathrm{Ca}^{2+}\right]$ is a decreasing function of spine volume, corresponding to the undercompensating scenario. If $\alpha>1$, then spine $\left[\mathrm{Ca}^{2+}\right]$ increases with spine volume, corresponding to the overcompensating scenario. For the representative simulations in Figures $2 C$ and 3, we set $\alpha$ equal to 1,0 , and 2 , respectively, for the compensating, undercompensating, and overcompensating scenarios. Synaptic weights are instantaneously updated at each timestep according to the $\mathrm{Ca}^{2+}$-dependent plasticity rule above.

For simulations described in Figure 3, all 500 synapses were initially set to approximately the same strength (Gaussian distributed with mean of $6 \mathrm{pA}$ and SD of $0.3 \mathrm{pA})$, and continuously stimulated at a low rate $(\sim 5 \mathrm{~Hz})$. A 
subset (50) of these synapses were then stimulated with either one or three bursts of 40 spikes at $80 \mathrm{~Hz}$. The interburst interval is $1000 \mathrm{~ms}$. We denote the mean strength of the stimulated synapses as $\bar{w}_{\text {stim }}(t)=\frac{\sum_{i=1}^{N_{\text {stim }}} w_{i}(t)}{N_{\text {stim }}}$. The drift rate, $D$ (see Fig. $3 B$ ), was the change in $\bar{w}_{\text {stim }}(t)$ over a time interval, $\Delta t$, divided by $\Delta t$, as follows:

$$
D=\left(\bar{w}_{\text {stim }}\left(t_{0}+\Delta t\right)-\bar{w}_{\text {stim }}\left(t_{0}\right)\right) / \Delta t .
$$

In Figure $3 B, D$ is plotted as a function of the initial mean strength, $\bar{w}_{\text {stim }}\left(t_{0}\right) . \Delta t$ here was $60 \mathrm{~s}(1 \mathrm{~min})$ of biological time.

Fokker-Planck model. The Fokker-Planck equation can be used to describe the time evolution of a probability density function (van Kampen, 1992). Here we use the form:

$$
\frac{\partial P\left(V_{\mathrm{sp}}, t\right)}{\partial t}=-\frac{\partial}{\partial V_{\mathrm{sp}}}\left[A\left(V_{\mathrm{sp}}\right) P\left(V_{\mathrm{sp}}, t\right)\right]+\frac{1}{2} \frac{\partial^{2}}{\partial V_{\mathrm{sp}}^{2}}\left[B^{\prime}\left(V_{\mathrm{sp}}\right) P\left(V_{\mathrm{sp}}, t\right)\right],
$$

where $P\left(V_{\mathrm{sp}}, t\right)$ is the time-dependent spine volume probability distribution, $V_{\text {sp }}$ is the spine head volume, and $A\left(V_{\mathrm{sp}}\right)$ and $B^{\prime}\left(V_{\mathrm{sp}}\right)$ are the drift and diffusion terms, respectively. We numerically evaluate $A$ and $B^{\prime}$ as follows. First, we use a discretized $\mathrm{Ca}^{2+}$ concentration probability distribution. In the implementation described here, the distribution is exponential. Qualitatively similar results were found with either uniform or log-normal distributions, or when a limit of $20 \mu \mathrm{M}$ is imposed on the amplitude of the exponential distribution, because of the saturation in the plasticity rule at high $\left[\mathrm{Ca}^{2+}\right]$. The $\mathrm{Ca}^{2+}$-dependent plasticity rule is then used to calculate the discrete probability distribution of plasticity jump sizes for a given spine volume, $Q\left(\Delta V_{\mathrm{sp}}\right)$. From this we calculate the average jump, $\left\langle\Delta V_{\mathrm{sp}}\right\rangle=\Sigma_{i} V_{\mathrm{sp}, i} Q^{i}$, and its mean square, $\left\langle\left(\Delta V_{\mathrm{sp}}\right)^{2}\right\rangle=\Sigma_{i}\left(V_{\mathrm{sp}, i}\right)^{2} Q_{\mathrm{i}}$. The average jump size as a function of spine volume gives the drift term, $A\left(V_{\mathrm{sp}}\right)$, while the mean square jump gives the diffusion term, $B\left(V_{\mathrm{sp}}\right)$ (van Kampen, 1992). To incorporate intrinsic fluctuations, we add a volumedependent term to the diffusion term, based on data demonstrating that the magnitude of spine size fluctuations increases linearly with spine head volume (parameters adapted from Yasumatsu et al., 2008). The final diffusion term, $B^{\prime}\left(V_{\mathrm{sp}}\right)$, is given by

$$
B^{\prime}\left(V_{\mathrm{sp}}\right)=B\left(V_{\mathrm{sp}}\right)+\left(2 \times 10^{-8}\right)\left(1+20 V_{\mathrm{sp}} \mu m^{-3}\right) .
$$

For simulation, we discretize the spine volume probability distribution, $P\left(V_{\mathrm{sp}}, t\right)$, at resolutions $\Delta t$ and $\Delta V$. We use the drift and diffusion terms to build a Markov transition matrix, $\boldsymbol{M}$, so that $P(t+\Delta t)=\Delta t \boldsymbol{M P}(t)$. It is important that $\Delta t$ and $\Delta V$ are small enough for the Fokker-Planck assumptions to hold. If we let $a_{i}=A_{i} / 2 \Delta V$ and $b_{i}=B_{i}^{\prime} / 2(\Delta V)^{2}$, then $\boldsymbol{M}$ is the tridiagonal matrix, as follows:

$$
\boldsymbol{M}=\left(\begin{array}{cccccc}
-a_{1}-b_{1} & -a_{2}+b_{2} & 0 & \ldots & 0 & 0 \\
a_{1}+b_{1} & -2 b_{2} & -a_{3}+b_{3} & \ldots & 0 & 0 \\
0 & a_{2}+b_{2} & -2 b_{3} & \ldots & 0 & 0 \\
\vdots & \vdots & \vdots & \ddots & \vdots & \vdots \\
0 & 0 & 0 & \ldots & -2 b_{n-1} & -a_{n}+b_{n} \\
0 & 0 & 0 & \ldots & a_{n-1}+b_{n-1} & a_{n}-b_{n}
\end{array}\right) .
$$

Because the columns of $\boldsymbol{M}$ sum to zero, it has at least one eigenvalue equal to zero. The eigenvector associated with the zero eigenvalue corresponds to the steady-state probability distribution (see Fig. 5).

For Figure 4, $A$ and $B$, left column (the compensating scenario), we tune the $\mathrm{Ca}^{2+}$ amplitude distribution so that depression is slightly more likely than potentiation. Hence, the synaptic strength probability distribution always drifts to the minimum strength, no matter what the initial strength is. For Figure 4, $C$ and $D$, we measure the time to steady state (the lifetime) as the time it takes for the median of the spine volume probability distribution to reach $\pm 20 \%$ of the steady-state median strength. We choose the median instead of the mean because the distributions were often bimodal, and graphing the median more clearly represented the simulation findings, although the results were qualitatively the same in either case. For Figure $4 E$, we initially set the synaptic strength to the minimum strength. We then define the probability of a spontaneous

\begin{tabular}{|c|c|c|}
\hline Symbol & Value & Parameter \\
\hline$C_{m}$ & $0.75 \mu \mathrm{F} / \mathrm{cm}^{2}$ & Membrane capacitance \\
\hline$R_{a}$ & $200 \Omega \mathrm{cm}$ & Axial resistivity \\
\hline $\bar{g}_{\text {leak }}$ & $2 \mathrm{pS} / \mu \mathrm{m}^{2}$ & Leak conductance density \\
\hline$e_{\text {leak }}$ & $-70 \mathrm{mV}$ & Leak reversal potential \\
\hline $\bar{g}_{\text {Cavsp }}$ & $300 \mathrm{ps} / \mu \mathrm{m}^{2}$ & Spine total CaV conductance density \\
\hline $\bar{g}_{\text {Cavd }}$ & $40 \mathrm{ps} / \mu \mathrm{m}^{2}$ & Dendrite total CaV conductance density \\
\hline eCa & $+10 \mathrm{mV}$ & $\mathrm{Ca}^{2+}$ reversal potential (Ohmic) \\
\hline$I_{\text {neck }}$ & $0.5 \mu \mathrm{m}$ & Spine neck length \\
\hline$d_{\text {neck }}$ & $0.1 \mu \mathrm{m}$ & Spine neck diameter \\
\hline$I_{\text {dend }}$ & $795.77 \mu \mathrm{m}$ & Dendrite length \\
\hline$d_{\text {dend }}$ & $2 \mu \mathrm{m}$ & Dendrite diameter \\
\hline$\tau_{\text {rise }}^{\mathrm{AMA}}$ & $0.18 \mathrm{~ms}$ & GluA rise time constant \\
\hline$\tau_{\text {decay }}^{\text {AMPA }}$ & $1.8 \mathrm{~ms}$ & GluA decay time constant \\
\hline$\tau_{\text {rise }}^{\mathrm{NMDA}}$ & $2 \mathrm{~ms}$ & GluN rise time constant \\
\hline$\tau_{\text {decay }}^{\mathrm{NMDA}}$ & $89 \mathrm{~ms}$ & GluN decay time constant \\
\hline$e_{\text {syn }}$ & $0 \mathrm{mV}$ & GluA and GluN reversal potential \\
\hline$\left[\mathrm{Ca}^{2+}\right]_{0}$ & $50 \mathrm{~nm}$ & Resting $\mathrm{Ca}^{2+}$ concentration \\
\hline$D$ & $2.2 \times 10^{-10} \mathrm{~m}^{2} / \mathrm{s}$ & $\mathrm{Ca}^{2+}$ diffusion rate constant \\
\hline$\beta_{\mathrm{sp}}$ & $0.8 \times 10^{-4} / \mathrm{s}$ & Spine extrusion rate \\
\hline$\beta_{\text {dend }}$ & $3.2 \times 10^{-4} / \mathrm{s}$ & Dendrite extrusion rate \\
\hline$k_{f}$ & $100 \times 10^{6} / \mathrm{M} / \mathrm{s}$ & Endogenous buffer forward binding rate \\
\hline$k_{b}$ & $500 / \mathrm{s}$ & Endogenous buffer backward binding rate \\
\hline$B_{\text {Tsp }}$ & $100 \mu \mathrm{m}$ & Spine total buffer concentration \\
\hline$B_{\text {Tdend }}$ & $500 \mu \mathrm{M}$ & Dendrite total buffer concentration \\
\hline$\eta_{p}$ & $1 \times 10^{-9} \mathrm{~s} / \mathrm{s}$ & Potentiation rate \\
\hline$\theta_{p}$ & $5.5 \mu \mathrm{M}$ & Offset for potentiation sigmoid \\
\hline$\sigma_{p}$ & $0.2 \mu \mathrm{m}$ & Slope for potentiation sigmoid \\
\hline$\eta_{d}$ & $5 \times 10^{-10} \mathrm{~S} / \mathrm{s}$ & Depression rate \\
\hline$\theta_{d}$ & $4 \mu \mathrm{M}$ & Offset for depression sigmoid \\
\hline$\sigma_{d}$ & $0.2 \mu \mathrm{M}$ & Slope for depression sigmoid \\
\hline
\end{tabular}
transition from the weak to strong stable strength as $p_{\text {flip }}=P\left(V_{\mathrm{sp}}>\right.$ $\left.V_{\text {max }} / 2\right)$ following a fixed simulation time, $t_{\text {flip }}$. Here $t_{\text {flip }}=5000 \mathrm{~s}$, but
Table 1. Biophysical model parameter values

varying this choice does not change the qualitative shape of the curve in Figure $4 E$.

Biophysical CA1 pyramidal neuron spine model. The biophysical spine model (Fig. 6A) includes both $\mathrm{Ca}^{2+}$ and electrical dynamics and consists of the following three compartments: a spherical head (volume range 0.01-0.3 $\mathrm{mm}^{3}$; Harris and Stevens, 1989); a cylindrical spine neck (diameter $0.1 \mu \mathrm{m}$, length $0.5 \mu \mathrm{m}$ ) (Harris and Stevens, 1989); and a singlecompartment cylindrical dendrite segment (diameter $2 \mu \mathrm{m}$, length $795.77 \mu \mathrm{m}$, surface area $5000 \mu \mathrm{m}^{2}$ ). The values of the model parameters are given in Table 1. The simulation code is available to download from the online database ModelDB (http://senselab.med.yale.edu/modeldb/).

The electrical component contained GluAs (AMPA receptors), GluNs, a leak conductance, and four different voltage-gated $\mathrm{Ca}^{2+}$ conductances: $\mathrm{Ca}_{\mathrm{v}} 3.1$ (T-type), $\mathrm{Ca}_{\mathrm{v}} 2.3$ (R-type), $\mathrm{Ca}_{\mathrm{v}} 1.2 / 1.3$ (L-type), and Ca 2.2 (N-type). These four $\mathrm{Ca}^{2+}$ channel types can account for the majority of $\mathrm{Ca}^{2+}$ influx through $\mathrm{Ca}^{2+}$ channels (CaVs) at hippocampal spines (Bloodgood and Sabatini, 2007). The time course of GluA and GluN glutamate binding was expressed as the dimensionless quantity, $G$, and modeled as the difference between two exponentials, $G_{\text {AMPA/NMDA }}=e^{-t / \tau_{\text {decay }}}-e^{-t / \tau_{\text {rise }}}$. The GluN model also contained a voltage-dependent $\mathrm{Mg}^{+}$block (Jahr and Stevens, 1990), so the total GluN conductance is as follows:

$$
g_{\mathrm{NMDA}}=\frac{\bar{g}_{\mathrm{NMDA}} \times G_{\mathrm{NMDA}}}{1+e^{-0.063 V_{m}} \frac{M g^{+}}{3.57}},
$$

where $\bar{g}_{\text {NMDA }}$ is the peak GluN conductance, $V_{m}$ is the spine membrane potential $(\mathrm{mV})$, and $\left[\mathrm{Mg}^{+}\right]$is the extracellular magnesium concentration (mM), here taken as $1 \mathrm{~mm}$ (Jahr and Stevens, 1990). The CaV models were adapted from the literature as follows: $\mathrm{Ca}_{\mathrm{v}} 3.1$ (T-type) from Traboulsie et al. (2007); $\mathrm{Ca}_{\mathrm{v}} 2.3$ (R-type) from Grunditz et al. (2008); $\mathrm{Ca}_{\mathrm{v}} 1.2 / 1.3$ (L-type) from Carlin et al. (2000); and $\mathrm{Ca}_{\mathrm{v}} 2.2$ (N-type) from Huang and Robinson (1998). The postsynaptic voltage was driven to the 
specified voltage by a tonic current stimulus to the dendrite, and activation of synaptic conductances depolarized the voltage even further. The three-compartment circuit was simulated using standard compartmental modeling methods (Segev and Koch, 1998).

The $\mathrm{Ca}^{2+}$ dynamics had parameters hand tuned to reproduce the data from Sabatini et al. (2002). $\mathrm{Ca}^{2+}$ entered the spine head through GluNs and $\mathrm{Ca}^{2+}$ channels, and was then buffered, extruded through membrane pumps, or diffused to the dendrite. The spine head $\mathrm{Ca}^{2+}$ concentration, $\left[\mathrm{Ca}^{2+}\right]_{\mathrm{sp}}(t)$, evolved as follows:

$$
\begin{aligned}
\frac{d\left[\mathrm{Ca}^{2+}\right]_{\mathrm{sp}}(t)}{d t}= & \frac{-I_{\mathrm{Ca}}}{z F V_{\mathrm{sp}}} \\
& -\left(\left[\mathrm{Ca}^{2+}\right]_{\mathrm{sp}}(t)-\left[\mathrm{Ca}^{2+}\right]_{0}\right) \frac{\beta_{\mathrm{sp}} S_{\mathrm{sp}}}{V_{\mathrm{sp}}} \\
& -D \frac{\left(\left[\mathrm{Ca}^{2+}\right]_{\mathrm{sp}}(t)-\left[\mathrm{Ca}^{2+}\right]_{\text {neck }}(t)\right) A_{\text {neck }}}{l_{\text {neck }} V_{\mathrm{sp}}} \\
& -k_{f}\left([B]_{\mathrm{sp}}(t)\left[\mathrm{Ca}^{2+}\right]_{\mathrm{sp}}(t)\right)+k_{b}\left([B]_{\mathrm{Tsp}}-[B]_{\mathrm{sp}}(t)\right) .
\end{aligned}
$$

The first term on the right-hand side represents $\mathrm{Ca}^{2+}$ influx; $I_{\mathrm{Ca}}$ (amperes $)$ is the total $\mathrm{Ca}^{2+}$ current influxing to the spine $\left(I_{\mathrm{Ca}}=I_{\mathrm{CaV}}+0.1 \times\right.$ $\left.I_{\mathrm{NMDA}}\right), z$ is the $\mathrm{Ca}^{2+}$ ionic charge, 2 , and $F$ is the Faraday constant. The second term represents extrusion through the membrane; $\left[\mathrm{Ca}^{2+}\right]_{0}$ is the resting $\mathrm{Ca}^{2+}$ concentration $(\mu \mathrm{M}), \beta_{\mathrm{sp}}$ is the extrusion rate $\left(\mu \mathrm{m} \cdot \mathrm{s}^{-1}\right)$, and $S_{\mathrm{sp}}$ is the spine head surface area $\left(\mu \mathrm{m}^{2}\right)$. The third term is diffusion through the spine neck according to Fick's law; $D$ is the $\mathrm{Ca}^{2+}$ cytoplasmic diffusion constant $\left(\mu \mathrm{m}^{2} / \mathrm{s}\right),\left[\mathrm{Ca}^{2+}\right]_{\text {neck }}$ is the spine neck $\mathrm{Ca}^{2+}$ concentration $(\mu \mathrm{M}), A_{\text {neck }}$ is the cross-sectional area of the spine neck $\left(\mu \mathrm{m}^{2}\right)$, and $l_{\text {neck }}$ is the length of the spine neck $(\mu \mathrm{m})$. The fourth and fifth terms represent $\mathrm{Ca}^{2+}$ binding and unbinding with endogenous buffer, respectively; $k_{f}$ and $k_{b}$ are the forward $\left(\mu \mathrm{M}^{-1} \mathrm{~s}^{-1}\right)$ and backward $\left(\mathrm{s}^{-1}\right) \mathrm{Ca}^{2+}$ buffer binding rate constants, respectively; $[\mathrm{B}]_{\mathrm{Tsp}}$ is the fixed total concentration of endogenous $\mathrm{Ca}^{2+}$ buffer $(\mu \mathrm{M})$; and $[\mathrm{B}]_{\mathrm{sp}}(t)$ is the dynamic concentration of unbound endogenous $\mathrm{Ca}^{2+}$ buffer $(\mu \mathrm{M})$. Analogous equations govern $\mathrm{Ca}^{2+}$ dynamics in the spine neck and dendrite.

The $\mathrm{Ca}^{2+}$-to- $\mathrm{Ca}^{2+}$ buffer reaction was modeled according to the kinetic equation $\left[\mathrm{Ca}^{2+}\right]_{\mathrm{sp}}+[\mathrm{B}]_{\mathrm{sp}} \leftrightarrow[\mathrm{BCa}]$, where $[B C a]$ is the concentration of $\mathrm{Ca}^{2+}$-bound buffer, $[B C a]=[B]_{\mathrm{Tsp}}-[B]_{\mathrm{sp}}$. The free buffer, $[B]_{\mathrm{sp}}$, evolves as

$$
\frac{d[B]_{\mathrm{sp}}}{d t}=-k_{f}\left([B]_{\mathrm{sp}}\left[\mathrm{Ca}^{2+}\right]_{\mathrm{sp}}\right)+k_{b}\left([B]_{\mathrm{Tsp}}-[B]_{\mathrm{sp}}\right) .
$$

In this model, the spine's endogenous buffer capacity, $\kappa_{\mathrm{E}}$, is as follows:

$$
\begin{aligned}
\kappa_{E} & =[B]_{\text {Tsp }}(t) k_{f} / k_{b} \\
& =100 \mu M \times 10^{8} M^{-1} s^{-1} / 500 s^{-1} \\
& =20,
\end{aligned}
$$

implying that $\sim 95 \%$ of the $\mathrm{Ca}^{2+}$ influx to the spine is buffered (Sabatini et al., 2002). The remainder of the $\mathrm{Ca}^{2+}$ is rapidly extruded, and only a small fraction $(<1 \%)$ diffuses to the dendrite (Sabatini et al., 2002). Hence, the spine neck plays only a negligible role in $\mathrm{Ca}^{2+}$ dynamics.

The peak GluA conductance, $\bar{g}_{\text {AMPA }}$, was proportional to spine head volume, $\bar{g}_{\mathrm{AMPA}}=5000 \mathrm{pS} / \mu \mathrm{m}^{3}$, while peak GluN conductance, $\bar{g}_{\mathrm{AMPA}}$, was independent of spine head volume, $\bar{g}_{\mathrm{NMDA}}=90 \mathrm{pS}$ (Fig. $6 \mathrm{~B}$ ). The GluNspine size relationship is crucial for the predictions of the biophysical model. We obtained qualitatively similar results if we assumed a GluN conductance that weakly increased with spine size $\left(\bar{g}_{\mathrm{NMDA}}=35+250 V_{\mathrm{sp}}\right)$ (Noguchi et al., 2005).

Although the rate of $\mathrm{Ca}^{2+}$ diffusion through the spine neck has been shown to be activity dependent, it appears to be regulated independently of spine head size (Bloodgood and Sabatini, 2005; Grunditz et al., 2008). EM studies also find that spine neck diameter (a key regulator of diffusion through the spine neck) has no correlation with spine head volume in hippocampus (Harris et al., 1992). This is in contrast to spine head size, which is tightly correlated with synaptic strength (Matsuzaki et al., 2001). It therefore seems unlikely that the rate of diffusion through the spine neck is also tightly scaled with synaptic strength. Hence, we do not investigate the implications of scaling of spine neck properties.
The stimulation protocol for Figure 6 was a burst of 100 synaptic inputs at $100 \mathrm{~Hz}$ with the postsynaptic voltage driven to the described potential by a tonic current stimulus to the dendrite.

Molecular-level MCell spine model. The model was adapted from Keller et al. (2008) and implemented with the MCell simulator (Stiles et al., 1996). The spine head is represented as a single cube with edge length scaled to achieve the desired volume. One side of the cube is chosen as the postsynaptic density (PSD) and contains 20 GluNs, independent of spine volume. GluNs were here represented as simple $\mathrm{Ca}^{2+}$ sources, which released $\mathrm{Ca}^{2+}$ ions into the spine at a fixed rate, governed by the $\mathrm{Mg}^{+}$ block voltage dependence (Jahr and Stevens, 1990). To simulate elevated postsynaptic activity during common synaptic plasticity protocols (Lee et al., 2009), the postsynaptic membrane potential is set to $-30 \mathrm{mV}$. The spine bulk contains the following three $\mathrm{Ca}^{2+}$ buffers: mobile calbindin (45 $\mu \mathrm{M})$; immobile calmodulin $(10 \mu \mathrm{M})$; and a generic fast immobile endogenous buffer $(5 \mu \mathrm{M})$. The spine membrane uniformly contains plasma membrane $\mathrm{Ca}^{2+}$ ATPase (PCMA) pumps, $\mathrm{Na}^{+}-\mathrm{Ca}^{2+}$ exchangers (NCXs), and a constant low-rate $\mathrm{Ca}^{2+}$ influx to maintain resting $\mathrm{Ca}^{2+}$ concentration (Keller et al., 2008). The base of the spine contains a square patch of membrane $0.15 \times 0.15 \mu \mathrm{m}^{2}$ that is transparent to $\mathrm{Ca}^{2+}$, modeling $\mathrm{Ca}^{2+}$ escape by diffusion through the spine neck to the dendrite.

The $\mathrm{Ca}^{2+}$ nanodomain signals are measured around a single $\mathrm{Ca}_{\mathrm{v}} 1$ (L-type) channel (Magee and Johnston, 1995) that is inserted into the center of the PSD. We measure instantaneous nanodomain $\mathrm{Ca}^{2+}$ concentrations by placing a transparent sampling cube of $20 \times 20 \times 20 \mathrm{~nm}$ size at the desired distance from the channel pore, counting the number of molecules in the box and dividing by the volume and Avagadro's number to get the molar concentration. We choose the L-type CaV because it is the only $\mathrm{CaV}$ that has been implicated in local postsynaptic $\mathrm{Ca}^{2+}$ signaling involved in synaptic plasticity (Yasuda et al., 2003; Lee et al., 2009). Nanodomains both form and disappear rapidly (within hundreds of microseconds) when the channel opens and closes, respectively, and are maximal in the steady state (Neher, 1998). Because these time scales are comparable to the mean channel open time of L-type channels ( $\sim 1 \mathrm{~ms}$ ) (Magee and Johnston, 1995), stochastic gating can have the effect of reducing the amplitude of nanodomain $\mathrm{Ca}^{2+}$ concentrations by cutting short the transient to steady state. This reduction in the amplitude of nanodomain $\mathrm{Ca}^{2+}$ concentrations from stochastic channel gating enhances the effect of spine size on nanodomain signals, because the relative magnitude of the bulk $\mathrm{Ca}^{2+}$ concentration (which the spine size directly influences) is greater than it would be compared with the steadystate nanodomain concentration.

\section{Results}

\section{The spine size- $\mathrm{Ca}^{2+}$ influx relationship is a critical factor in synaptic plasticity}

How does the relationship between spine size and $\mathrm{Ca}^{2+}$ influx mechanisms affect synaptic plasticity? To address this question, we first consider a single dendritic spine attached to its parent dendrite. We use this generic model to introduce a general framework, which can then be applied to any spine.

Fast EPSCs from synapses are primarily mediated by spine GluAs. The number and state of synaptic GluAs are important factors that determine the strength of a synapse. Throughout this study, we assume that synaptic GluA number, and therefore synaptic efficacy, is proportional to spine head volume (Fig. 1 Aiii) (Takumi et al., 1999; Matsuzaki et al., 2001; Ganeshina et al., 2004; Noguchi et al., 2005).

$\mathrm{Ca}^{2+}$ influx to spines arises primarily from GluNs and CaVs, and is determined by both presynaptic and postsynaptic activity patterns. Intracellular $\left[\mathrm{Ca}^{2+}\right]$ changes of sufficient amplitude can trigger synaptic plasticity (Lynch et al., 1983). We assume that the $\mathrm{Ca}^{2+}$ concentration within the spine directly determines both the magnitude and polarity of synaptic plasticity (Bienenstock et al., 1982; Bear et al., 1987; Lisman, 1989; Cummings et al., 1996; Hansel et al., 1997; Yang et al., 1999; Cho et al., 2001; Cormier et al., 2001; Shouval et al., 2002; Ismailov et al., 2004). 
Low or baseline spine $\left[\mathrm{Ca}^{2+}\right]$ causes no change in synaptic strength. Intermediate $\left[\mathrm{Ca}^{2+}\right]$ triggers synaptic depression, while high $\left[\mathrm{Ca}^{2+}\right]$ triggers synaptic potentiation (Fig. 1Aii), with corresponding changes in spine size.

Given these assumptions, there are three possible scenarios by which spine plasticity can influence spine $\left[\mathrm{Ca}^{2+}\right]$ changes in response to stimuli (Fig. $1 B-$ $D)$. First, $\mathrm{Ca}^{2+}$ concentration in a spine may be independent of spine volume (Fig. $1 B-D$, left column). This scenario can only be achieved if $\mathrm{Ca}^{2+}$ influx mechanisms are continuously scaled during synaptic plasticity to exactly counter changes in spine volume (Fig. $1 \mathrm{Bi}, \mathrm{Ci}$ ). We refer to this case as the compensating scenario. In this case, because $\mathrm{Ca}^{2+}$ concentration does not depend upon spine size (Fig. $1 D i$ ), the direction and magnitude of synaptic plasticity is dictated only by neuronal activity and not by synaptic strength. Although possible in principle, exact compensation is perhaps unlikely because it requires the rapid and precise tuning of multiple spine $\mathrm{Ca}^{2+}$ properties. For example, to compensate $\mathrm{Ca}^{2+}$ influx for changes in spine volume, the spine would need to scale the density of its $\mathrm{Ca}^{2+}$ permeable channels and receptors proportional to $r^{3 / 2}$ (where $r$ is the spine head radius) (see Materials and Methods).

Second, when spines increase in size, there might not be a corresponding increase in the number of $\mathrm{Ca}^{2+}$-permeable channels and receptors. We refer to this case as the undercompensating scenario (Fig. $1 B-D$, center column). For example, the number of $\mathrm{Ca}^{2+}$-permeable channels might be independent of spine head volume (Fig. 1Cii). In this case, small spines will experience greater $\left[\mathrm{Ca}^{2+}\right]$ transients than large spines (Fig. 1Dii). This also applies to the case where $\mathrm{Ca}^{2+}$ influx is proportional to spine surface area (see Materials and Methods). In the undercompensating scenario, a single stimulus might cause a $\left[\mathrm{Ca}^{2+}\right]$ change sufficient to trigger potentiation at small spines, but might cause a smaller $\left[\mathrm{Ca}^{2+}\right]$ change that triggers only depression at large spines. Very large spines will have $\left[\mathrm{Ca}^{2+}\right]$ changes too dilute to trigger synaptic plasticity at all (Fig. 1 Dii, spines $\left.>0.2 \mu \mathrm{m}^{3}\right)$. Hence, undercompensation makes strong synapses on very large spines immune to synaptic plasticity from ongoing neural activity.

Third, when spines increase in size the gain in their number of $\mathrm{Ca}^{2+}$-permeable channels might be greater than that required for exact compensation. We refer to this situation as the overcompensating scenario (Fig. $1 B-D$, right column). For example, a spine might double its volume following synaptic potentiation while trebling its number of GluNs. In this case, large spines
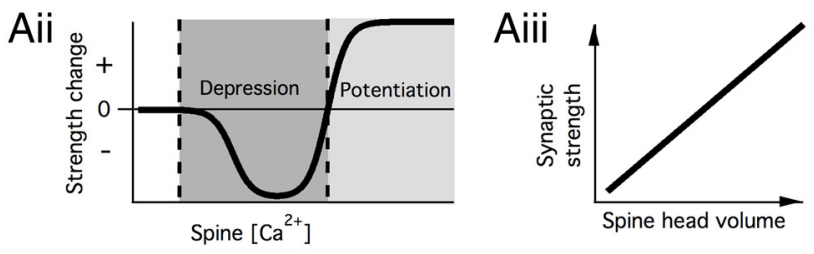

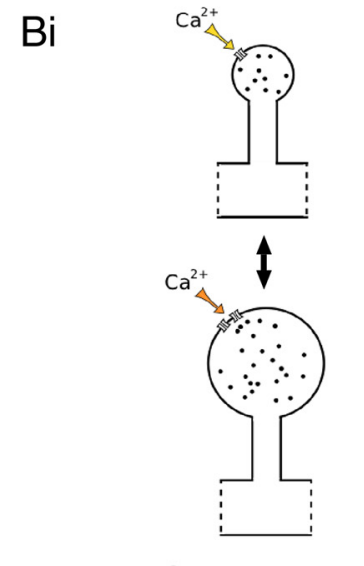

Compensating
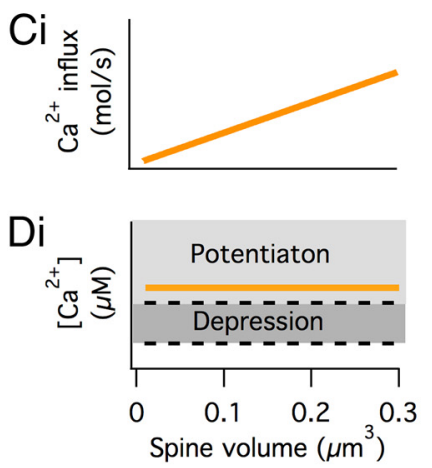

Bii

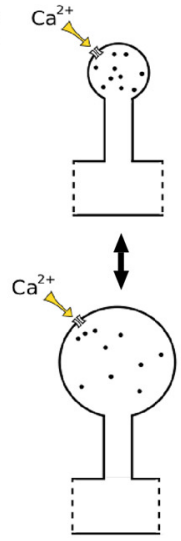

Undercompensating
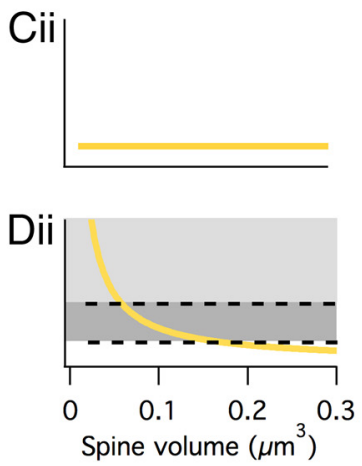

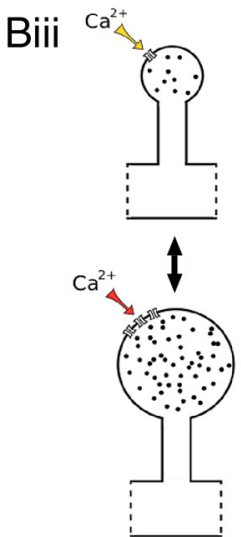

Overcompensating
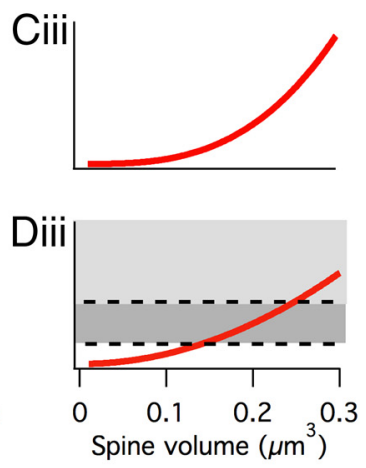

Figure 1. The relationship between spine size and $\mathrm{Ca}^{2+}$ influx falls into one of three different scenarios. $\boldsymbol{A i}$, The synaptic plasticity cascade. Coincident presynaptic and postsynaptic activity triggers postsynaptic $\mathrm{Ca}^{2+}$ signals, which are shaped by dendritic spine properties. $\mathrm{Ca}^{2+}$ signals trigger kinase- and phosphatase-based molecular cascades, resulting in long-term potentiation/depression. Aii, $\mathrm{Ca}^{2+}$-dependent synaptic plasticity rule. The change in synaptic strength as a function of spine $\left[\mathrm{Ca}^{2+}\right]$. There is a moderate threshold for depression and a higher threshold for potentiation. Aiii, Spine size is assumed to be proportional to synaptic strength, in accordance with experimental data (Matsuzaki et al., 2001). Bi, $\mathrm{Ca}^{2+}$ influx might exactly compensate for changes in spine volume so that $\left[\mathrm{Ca}^{2+}\right]$ is independent of spine size. Bii, $\mathrm{Ca}^{2+}$ influx might undercompensate for changes in spine volume so that large spines have lower amplitude $\left[\mathrm{Ca}^{2+}\right]$ transients than small spines. Biii, $\mathrm{Ca}^{2+}$ influx might overcompensate for changes in spine volume so that large spines have higher-amplitude $\left[\mathrm{Ca}^{2+}\right]$ transients than small spines. $\mathbf{C}, \mathbf{D}$, The absolute amount of $\mathrm{Ca}^{2+}$ influx $(\boldsymbol{C})$ and $\mathrm{Ca}^{2+}$ concentration change (D) following a plasticity-inducing stimulus, as a function of spine volume. $\mathbf{C i}$, In the compensating scenario, $\mathrm{Ca}^{2+}$ influx is proportional to spine volume. $\mathbf{D i}$, This leads to a volume-independent $\left[\mathrm{Ca}^{2+}\right]$. Hence, a given stimulus causes the same synaptic plasticity for spines of all sizes. Cii, In the undercompensating scenario, $\mathrm{Ca}^{2+}$ influx is sublinear with spine volume. Dii, This leads to large spines experiencing lower $\left[\mathrm{Ca}^{2+}\right]$ than small spines. Hence, a given stimulus can cause potentiation at small spines, depression at medium-sized spines, and no change in synaptic strength at large spines. Ciii, In the overcompensating scenario, $\mathrm{Ca}^{2+}$ influx is superlinear with spine volume. Diii, This leads to large spines experiencing higher $\left[\mathrm{Ca}^{2+}\right]$ than small spines. Hence, a given stimulus can cause potentiation at large spines, depression at medium-sized spines, and no synaptic strength change at small spines.

experience greater-amplitude $\left[\mathrm{Ca}^{2+}\right]$ transients from synaptic stimulation than small spines (Fig. 1Diii). Hence, according to the $\mathrm{Ca}^{2+}$-dependent plasticity rule, overcompensation makes strong synapses on large spines more susceptible to potentiation, and makes weak synapses on small spines more susceptible to depression (Fig. 1 Diii).

In summary, one of the three possible $\mathrm{Ca}^{2+}$ influx scenarios must occur when spine volume is changed during synaptic plasticity: compensation, undercompensation, or overcompensa- 


\section{Compensating}

$\mathrm{Ai}$

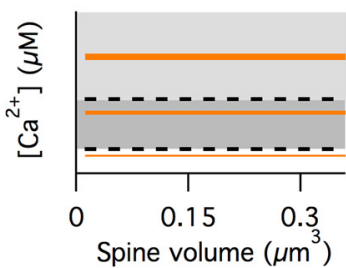

$\mathrm{Bi}$
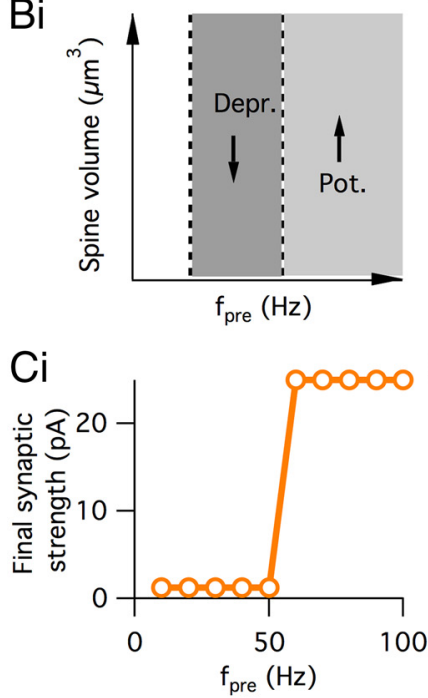

Cii

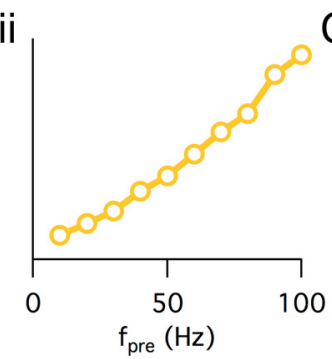

Undercompensating

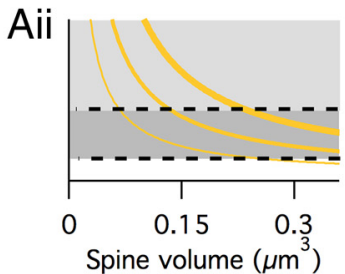

Overcompensating

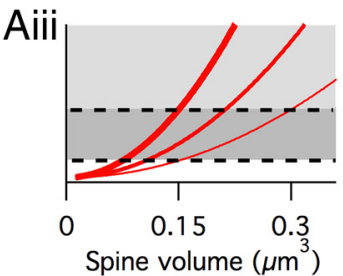

$\mathrm{Bii}$

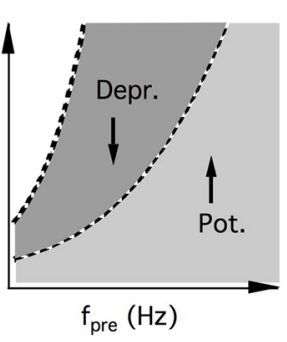

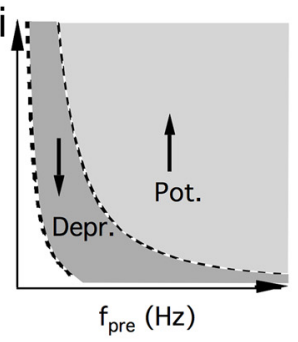

Biii

Figure 2. The effect of stimulus intensity on the direction of synaptic plasticity for the three different scenarios. $\boldsymbol{A}$, Increasing the stimulus intensity (from thinner to thicker lines) increases the $\mathrm{Ca}^{2+}$ influx across all synaptic strengths in all three cases. Ai, For the compensating rule, the direction of synaptic plasticity is dependent on stimulus intensity, but not on synaptic strength. Aii, For the undercompensating rule, each stimulus intensity leads to a corresponding stable strength (intersection of orange curves with upper dashed line). Above the stable strength, synapses are depressed, while below the stable strength, synapses are potentiated. Stronger stimuli shift the stable point to greater synaptic strengths. Aiii, For the overcompensating rule, each stimulus intensity has a corresponding unstable threshold strength (intersection of red curves with the upper dashed line). Above the threshold synapses are potentiated, while below the threshold synapses are depressed. Stronger stimuli shift the unstable threshold to weaker strengths. $\boldsymbol{B}$, Plasticity direction as a function of both stimulus strength (horizontal axis) and spine volume (vertical axis). Dashed black curves indicate thresholds for LTD and LTP. Arrows indicate the direction of change of synaptic strength. $\boldsymbol{C}$, Final synaptic strengths on an integrate-and-fire neuron following prolonged Poisson stimulation, as a function of presynaptic firing rate. $\mathbf{C}$, Compensation results in synapses eventually drifting to their maximum or minimum strength, depending on their stimulation rate. Cii, Undercompensation yields synapses that represent the stimulation rate as a continuous variable. Ciii, 0 vercompensation leads to either maximal or minimal strengths, but whether a synapse ends up strong or weak depends not only on the stimulus rate, but also on the initial synaptic strength. Above the separatrix (dashed gray curve), the synapse will potentiate, but below it will depress. Depr., Depression; Pot., potentiation.

tion. The scenario that occurs is determined by the relationship between spine volume and its number of $\mathrm{Ca}^{2+}$-permeable channels (the precise conditions for each scenario are described in Materials and Methods). It is not clear a priori which of the three scenarios applies to biological synapses.

\section{Undercompensation and overcompensation change the rules of synaptic plasticity compared with the compensating} scenario

How do the three spine $\mathrm{Ca}^{2+}$-handling scenarios affect long-term synaptic strength dynamics? To address this question, we take the spine model from Figure 1, but now consider what happens as the stimulus intensity is varied.

In general, an increase in the intensity of either a presynaptic or postsynaptic stimulus leads to an increase of $\mathrm{Ca}^{2+}$ influx to the spine. For example, elevating the presynaptic firing rate causes increased release of glutamate, which can then bind to GluAs and GluNs, causing increased local postsynaptic depolarization, and a consequent increase in spine $\mathrm{Ca}^{2+}$ influx through GluNs and CaVs. Similarly, increased firing of postsynaptic action potentials leads to depolarization at the synapse and an increase of spine $\mathrm{Ca}^{2+}$ influx through GluNs and CaVs. In Figure $2 \mathrm{~A}$, we plot spine $\left[\mathrm{Ca}^{2+}\right]$ as a function of spine volume for the three scenarios of exact compensation, undercompensation, and overcompensation (Fig. 2Ai,Aii,Aiii, respectively). The thin-to-thick curves indicate $\left[\mathrm{Ca}^{2+}\right]$ in spines for stimuli of increasing intensity. In spines of all sizes and in all three scenarios, increasing stimulus intensity always increases spine $\left[\mathrm{Ca}^{2+}\right]$. Importantly however, the consequences of varying stimulus intensity for synaptic plasticity differ between the scenarios. We will consider each scenario individually below.

Because spine $\left[\mathrm{Ca}^{2+}\right]$ depends on two different factors (stimulus intensity and spine volume), the direction of synaptic plasticity triggered by a stimulus will also depend on these two factors. To illustrate this point, we plot the direction of synaptic change as a function of both spine volume and stimulus intensity - presynaptic firing rate in this case-for each scenario (Fig. $2 B$ ). In the compensating scenario, the direction of synaptic plasticity is independent of spine volume (Fig. $2 \mathrm{Bi}$ ). In contrast, in both the undercompensating (Fig. 2 Bii) and overcompensating (Fig. 2 Biii) scenarios the direction of synaptic plasticity depends on spine volume.

To explore how these mechanisms work in practice, we simulate an integrate-and-fire neuron with synapses that obey compensating, undercompensating, or overcompensating plasticity rules (see Materials and Methods). We stimulate all synapses on the neuron with Poisson spike trains of a given rate and observe how the synaptic strengths evolve. Eventually, synaptic strengths settle to steady-state values (Fig. 2C). We measure these steady-state synaptic strength values for a range of different stimulus intensities (presynaptic firing rates). Now we discuss the three scenarios in turn.

If synapses exactly compensate their $\mathrm{Ca}^{2+}$ influx following spine size changes, then the direction of a plasticity event is determined only by stimulus intensity and not by spine volume (Fig. $2 A i, B i$ ). Weak activity causes no change, intermediate activity causes depression, and high activity causes potentiation (Fig. $2 A i$, thin, medium, and thick lines, respectively). With repeated stimulation, a compensating synapse will continue to potentiate or depress without limit because there is no inherent mechanism in the plasticity rule to provide an upper or lower limit to its strength. In our simulations, we impose hard upper and lower limits on synaptic strength to avoid unphysiologically strong or weak synapses (25 and 5 pA, respectively). Following repeated 
stimulation synaptic strengths converge to the imposed maximum or minimum strengths. Whether a synapse settles to the maximum or minimum strength depends only on its stimulus intensity, and not on its initial synaptic strength. Hence, exact compensation does not endow any particular initial synaptic strength with extra stability over other strengths. Therefore, while in principle exactly compensating synapses could initially store information as a continuous variable, if potentiation and depression are not finely balanced, they will eventually lose this information by drifting to their maximum or minimum strengths (Fig. 2Ci) (Fusi and Senn, 2006; Fusi and Abbott, 2007).

In the undercompensating scenario, because a plasticity-inducing stimulus triggers potentiation at weak synapses but depression at strong synapses, it drives synapses toward a stable target strength at the crossover point between potentiation and depression (Fig. 2 Aii, intersection of curve with the upper dashed line). Hence, repeating the same plasticity-inducing stimulus at an undercompensating synapse will eventually saturate plasticity at a fixed synaptic strength. Importantly, because increasing the stimulus intensity increases $\left[\mathrm{Ca}^{2+}\right]$ in synapses of all strengths, stronger stimuli to undercompensating synapses shift the stable target strength to greater values (Fig. 2 Aii, thin to thick lines), as observed experimentally (McNaughton et al., 1978). The steady-state synaptic strength is a monotonically increasing function of stimulus intensity (Fig. 2Cii). Hence, undercompensating synapses can store information as a continuous variable. The plasticity rule also naturally regulates synaptic strength so that no externally imposed limits on synaptic strength are necessary.

In the case of overcompensation, a plasticity-inducing stimulus will depress weak synapses but potentiate strong synapses (Fig. 2 Aiii, Biii). Hence, all stimuli drive synapses toward either their minimum or maximum limits. As for the compensating case, these minimum and maximum strengths must be imposed by some additional mechanism. Whether a given synapse potentiates or depresses depends on whether its initial synaptic strength is greater or less than a certain threshold [Fig. 2 Biii,Ciii (dotted gray line)]. This threshold depends on stimulus intensity such that stronger stimuli lower the threshold (Fig. 2 Biii,Ciii). In this scheme, the synapse is effectively binary because only the minimum and maximum synaptic strengths are stable. Although, in general, binary synapses cannot store as much information as multistate synapses (Barrett and van Rossum, 2008), binary storage is robust because small, undesired changes in synaptic strength are automatically corrected, and random transitions between the strong and weak states are unlikely (Petersen et al., 1998).

In summary, we find that the $\mathrm{Ca}^{2+}$ influx to spine size scenario crucially determines the long-term dynamics of synaptic strength and the form of synaptic information storage. Compensation leads to binary information storage, which is not robust; undercompensation leads to robust continuous-variable information storage; and overcompensation leads to robust binary information storage.

\section{Spine plasticity determines the influence of ongoing neural activity on synaptic strength stability}

Ongoing neural activity might degrade stored memories by occasionally causing spine $\left[\mathrm{Ca}^{2+}\right]$ transients large enough to trigger synaptic plasticity. We therefore examine whether the relationship between spine size and $\mathrm{Ca}^{2+}$ influx affects memory storage in the presence of ongoing neural activity. We compare how well each of the three scenarios allow information about a previous activity pattern to be stored in the synaptic strengths of an integrate-and-fire model neuron, similar to that presented in Figure 2C. We consider a neuron with 500 synapses driven by Poisson inputs that are tonically active at a rate just sufficient to keep the postsynaptic neuron active (both the prefiring and postfiring rates are $\sim 5 \mathrm{~Hz}$ ). To simulate weak and strong memory events, we subject 50 of the synapses to either one or three brief high-frequency trains of input, respectively (see Materials and Methods). These highfrequency input events potentiate the stimulated synapses. After the input events, synapses are left to evolve their strengths in the presence of baseline neural activity. Because the baseline presynaptic inputs arrive randomly in time (but with a fixed average firing rate), occasionally multiple inputs arrive simultaneously by chance. If they are of large enough amplitude, these chance events trigger spontaneous synaptic plasticity. These spontaneous synaptic strength changes will accumulate over time, and might eventually "wash out" the potentiation initially induced by the high-frequency stimulus. The stability of the memory depends on both the magnitude of the initial high-frequency stimulus $(1 \times$ or $3 \times)$ and on the $\mathrm{Ca}^{2+}$ influx to spine size scenario. We examine each scenario in turn. 
Compensating Undercompensating Overcompensating
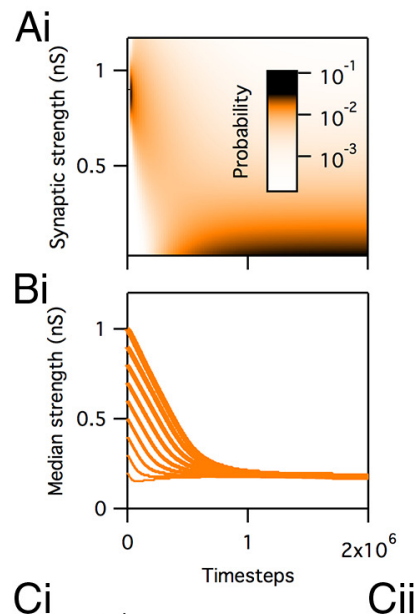

Aii

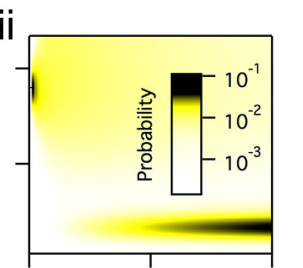

Bii

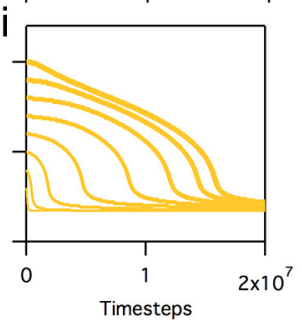

Aiii

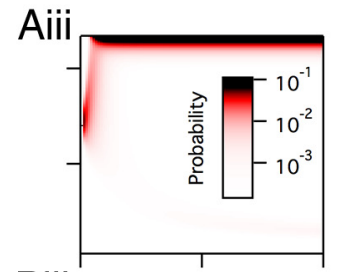

Biii
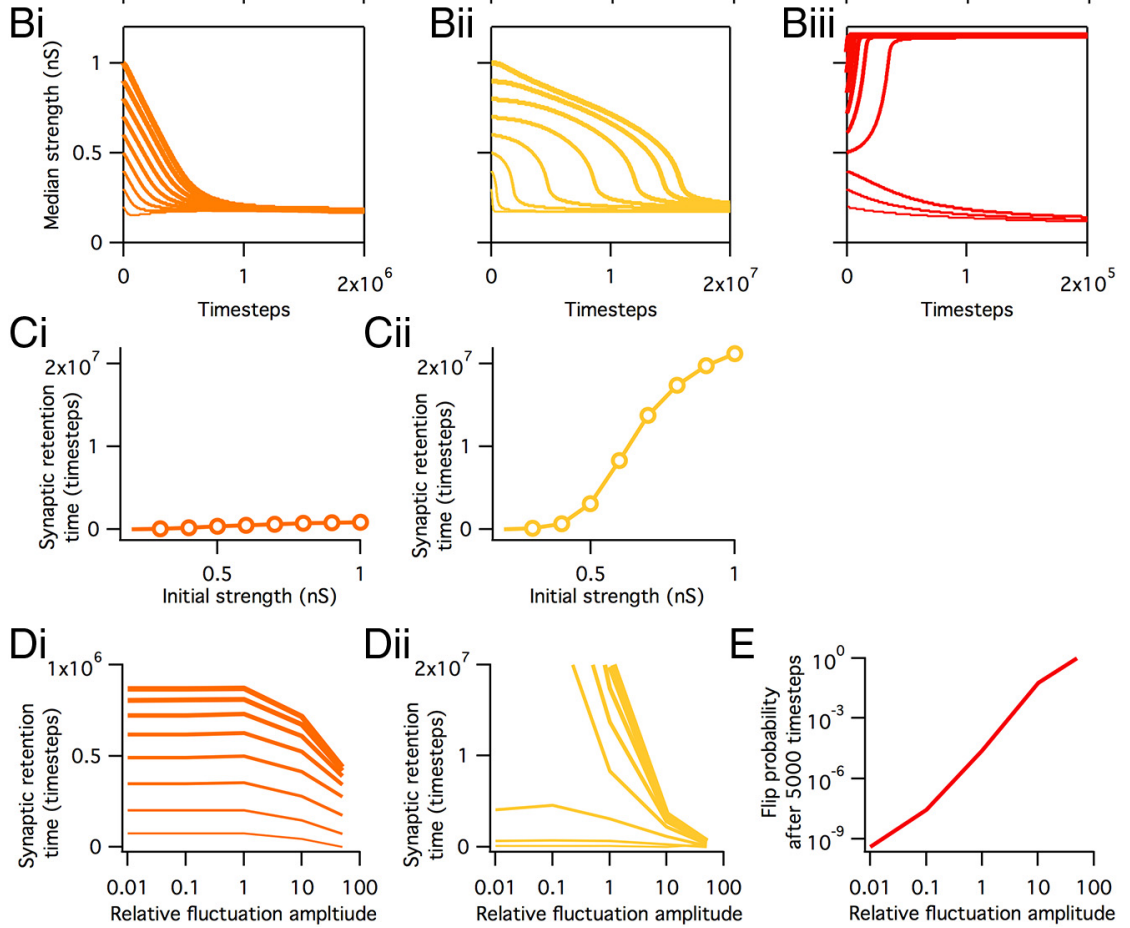

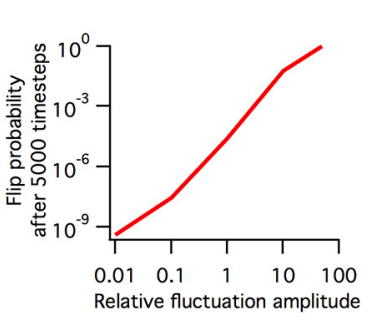

Figure 4. The lifetime of undercompensating and overcompensating synapses in the presence of intrinsic fluctuations is substantially longer than for compensating synapses in a Fokker-Planck model. Ai-Aiii, Synaptic strength probability distributions over time for the compensating (Ai), undercompensating (Aii), and overcompensating (Aiiii) scenarios. Darker color indicates higher probability. A synaptic plasticity event is simulated by initializing the synapse at a particular strength. The strength of the synapse then drifts probabilistically over time toward the steady-state strength. Note different time scales on the $x$-axis of each panel. Bi-Biii, Decay of median synaptic strength for the three learning rules for a range of different initial strength synapses. $\mathbf{C}$, Cii, The synaptic retention time for the compensating $(\boldsymbol{C} i)$ and undercompensating (Cii) learning rules as a function of initial synaptic strength. Undercompensation leads to very stable large spines, but their retention time is ultimately limited by intrinsic fluctuations. Di, Dii, Synaptic retention time for compensating (Di) and undercompensating (Dii) synapses of increasing initial strength (thin to thick lines) as a function of relative amplitude of fluctuations. Note differences in the time-scale axis between $\boldsymbol{D i}$ and Dii. E, Probability of overcompensating synapse spontaneously transitioning from lower to upper stable strength as a function of relative fluctuation amplitude.

In the exactly compensating scenario, in the presence of baseline activity the synaptic strengths drift in a direction determined by the ratio of the total amount of spontaneous potentiation to total spontaneous depression. Because in this example baseline activity triggers more depression than potentiation, all synapses experience net depression over time (Fig. 3Ai). Synapses drift toward their minimum strength $(5 \mathrm{pA})$. Importantly, the rate and direction of drift are independent of synaptic strength, such that no particular initial synaptic strength is more stable than any other (Fig. 3Bi).

In contrast, for the undercompensating scenario strong synapses stay potentiated for the duration of the simulation, because the large volumes of their spines dilutes $\left[\mathrm{Ca}^{2+}\right]$ transients so that spontaneous depression is rarely triggered. At the same time, intermediate strength synapses are occasionally spontaneously depressed, causing their synaptic strengths to eventually drift back to baseline (Fig. 3Aii). The rate of drift is slower for strong synapses than for weak synapses (Fig. 3Bii). In this way, under- compensating synapses lead to the persistence of strong synapses, and hence protects strong memory traces from plasticity due to ongoing activity.

In the overcompensating scenario, the behavior of synapses is qualitatively different from the two previous cases (Fig. 3Aiii, Biii). After initial potentiation, strong synapses are continually potentiated by ongoing activity until reaching the maximum strength $(25 \mathrm{pA})$, because overcompensation makes larger spines have higher-amplitude $\left[\mathrm{Ca}^{2+}\right]$ transients and therefore more susceptible to potentiation. Once these synapses reach the maximum strength they are fixed there indefinitely. Weaker synapses, in contrast, are subject to net depression and drift back toward baseline strength. The net drift from spontaneous potentiation and depression is positive for strong synapses and negative for weak synapses (Fig. 3Biii). Thus, overcompensating synapses threshold memory events into two categories: either strong and persistent or weak and transient.

\section{Spine plasticity increases synaptic lifetimes in the presence of intrinsic fluctuations}

In addition to spontaneous synaptic plasticity due to ongoing neural activity, dendritic spines exhibit intrinsic fluctuations in their size (Yasumatsu et al., 2008; Minerbi et al., 2009; Loewenstein et al., 2011), while postsynaptic densities demonstrate intrinsic fluctuations in both their size and shape (Mysore et al., 2007; Blanpied et al., 2008). Because these fluctuations are thought to be random (Yasumatsu et al., 2008), they may over time corrupt information encoded in the synaptic strength. To examine the impact of these fluctuations, we use a reduced mathematical model, based on the FokkerPlanck equation, where synaptic strength is described by a probability distribution. The intrinsic fluctuations have a diffusive effect on this probability distribution. We set the amplitude of intrinsic fluctuations to scale proportionally to spine size (Minerbi et al., 2009). Together, the activity-dependent plasticity and intrinsic fluctuations probabilistically determine the synapse's evolution (see Materials and Methods). This abstract model's key strength over more detailed models is that it contains only a small number of free parameters that are all based on clear assumptions. It therefore allows general properties to be established that would be difficult to uncover using either a biophysical or integrate-and-fire model neuron.

Using this model, we change the strength of a single synapse and then follow the time evolution of its synaptic strength probability distribution when the synapse is subject to $\left[\mathrm{Ca}^{2+}\right]$ transients from ongoing neural activity (Fig. $4 A$ ). We measure how long the synapse takes to equilibrate back to its baseline strength (Fig. 4). Once this equilibrium has been reached, any information 
stored in the synaptic strength is lost because it has become indistinguishable from other synapses. We measure the retention time as a function of initial synaptic strength for each of the three spine $\mathrm{Ca}^{2+}$-handling scenarios in the presence of intrinsic spine size fluctuations.

For the compensating scenario, the rate of drift toward equilibrium is independent of synaptic strength (Fig. 4Bi, parallel curves). As a result, the retention time of compensating synapses is poor, but scales linearly with initial strength (Fig. 4Ci). When the amplitude of intrinsic fluctuations is increased, retention time is decreased. The effect is similar for synapses of all strengths (Fig. $4 \mathrm{Di}$ ).

In contrast to the compensating scenario, in the undercompensating scenario the rate of decay of a potentiated synapse depends nonlinearly on its strength. All synapses eventually drift toward a single stable strength, but, because strong synapses are less susceptible to plasticity, they decay more slowly than weak synapses (Fig. 4 Bii,Cii). This relationship saturates only for very strong synapses where plasticity events are uncommon and intrinsic fluctuations begin to dominate (Fig. 4Cii). As we increase the amplitude of intrinsic fluctuations to extremely large values (Fig. 4Dii), synaptic retention time drops dramatically for synapses of all strength, because fluctuations are so large that they corrupt the synapse's ability to remain potentiated. Importantly, however, the retention time of undercompensating synapses is always greater than that of compensating synapses (Fig. 4, compare $D i$, Dii, time scales).

The overcompensating scenario causes the synaptic probability distribution to drift either to the minimum or maximum limit, depending both on neural activity and initial strength (Fig. 4Aiii,Biii). Small excursions from the stable minimum or maximum strengths quickly disappear. If intrinsic fluctuations are small, the synapse persists at the stable strengths indefinitely. If fluctuations are large, random transitions between the strong and weak states become likely. As a result, memory storage would be degraded (Fig. 4E).

These results demonstrate that the effect of intrinsic fluctuations on compensating synapses is independent of their size. In contrast, undercompensation reduces the sensitivity of large spines to intrinsic fluctuations, and overcompensation stabilizes spines that are already close to their maximum or minimum size. Thus, imperfect matching of $\mathrm{Ca}^{2+}$ dynamics and spine size may facilitate long-term storage of information by dendritic spines in the presence of intrinsic fluctuations.

\section{Undercompensation reproduces experimental synaptic strength distributions}

The shape of synaptic strength distributions can provide clues to the form of synaptic memory storage (Barbour et al., 2007). However, the consequences of spine structural dynamics for synaptic strength distributions are unknown. The synaptic strength distributions measured from hippocampus, neocortex, and cerebellum appear qualitatively similar (Barbour et al., 2007). They are typically continuous, unimodal, and have a peak at a nonzero strength and a long tail at high strengths. Although some electrophysiological and light microscopy techniques have noise amplitudes that make them likely to underestimate the prevalence of weak synapses, we note that the qualitative feature of a unimodal distribu- tion with nonzero peak is also reproduced by studies employing electron microscopy, where image resolution is beyond that necessary to unambiguously measure synapses of all size (Harris and Stevens, 1989; Schikorski and Stevens, 1997; Arellano et al., 2007; Mishchenko et al., 2010).

To determine whether any of the three spine size-to- $\mathrm{Ca}^{2+}$ influx scenarios described above could account for the in vivo distribution of spine sizes or synaptic strengths, we calculate equilibrium synaptic strength distributions reached after prolonged stimulation directly from the Fokker-Planck model introduced above (see Materials and Methods). The three scenarios lead to qualitatively different synaptic strength distributions (Fig. 5). Only the undercompensating case predicts a unimodal synaptic strength distribution with a central peak. If synapses compensate or overcompensate, strength distributions are either unimodal or bimodal with peaks at the minimum and/or maximum strengths but not at intermediate strengths. Hence, the undercompensating scenario is the most consistent with experimental findings.

\section{A dynamic biophysical hippocampal spine model predicts stable and undercompensating synapses}

It is not clear whether biological dendritic spines follow the compensating, undercompensating, or overcompensating scenario. To address this issue, we construct a dynamic model of a CA1 pyramidal neuron spine using available physiological data on spine morphology, and the number and type of spine ion channels and synaptic receptors.

Dendritic spine $\mathrm{Ca}^{2+}$ handling has been intensively studied in CA1 pyramidal neurons. The main source of $\mathrm{Ca}^{2+}$ influx from synaptic activation is GluNs (Sabatini et al., 2002). GluN number has been found to be either independent or weakly dependent on spine volume (Takumi et al., 1999; Racca et al., 2000; Nimchinsky et al., 2004; Noguchi et al., 2005; Sobczyk et al., 2005). In the simulations we describe here, we assume GluN conductance to be independent of spine volume, but similar results are obtained if GluN number is weakly dependent on spine volume (data not shown). In contrast, GluA conductance is directly proportional to spine volume (Nusser et al., 1998; Takumi et al., 1999; Matsuzaki et al., 2001; Noguchi et al., 2005) (Fig. 6B) (see Materials and Methods). In the spine model, we also include four types of voltage-gated $\mathrm{Ca}^{2+}$ channels: $\mathrm{Ca}_{\mathrm{v}} 3.1$ (T-type), $\mathrm{Ca}_{\mathrm{v}} 2.3$ (R-type), $\mathrm{Ca}_{\mathrm{v}}$ 1.2/1.3 (L-type), and $\mathrm{Ca}_{\mathrm{v}} 2.2$ (N-type). Together, these channels can account for $\mathrm{Ca}^{2+}$ influx through $\mathrm{CaV}$ s to hippocampal spines (Bloodgood and Sabatini, 2007). 
A
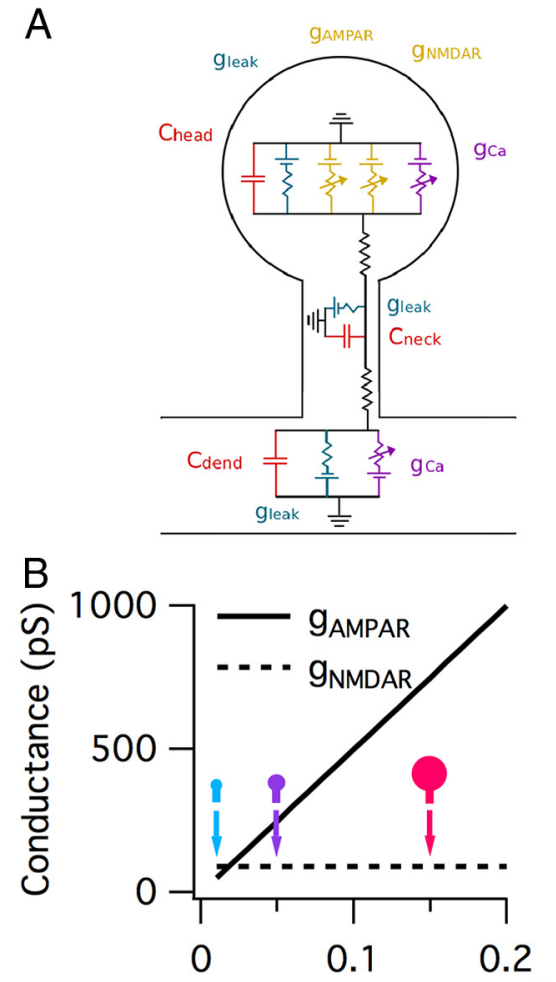

Spine head volume $\left(\mu \mathrm{m}^{3}\right)$
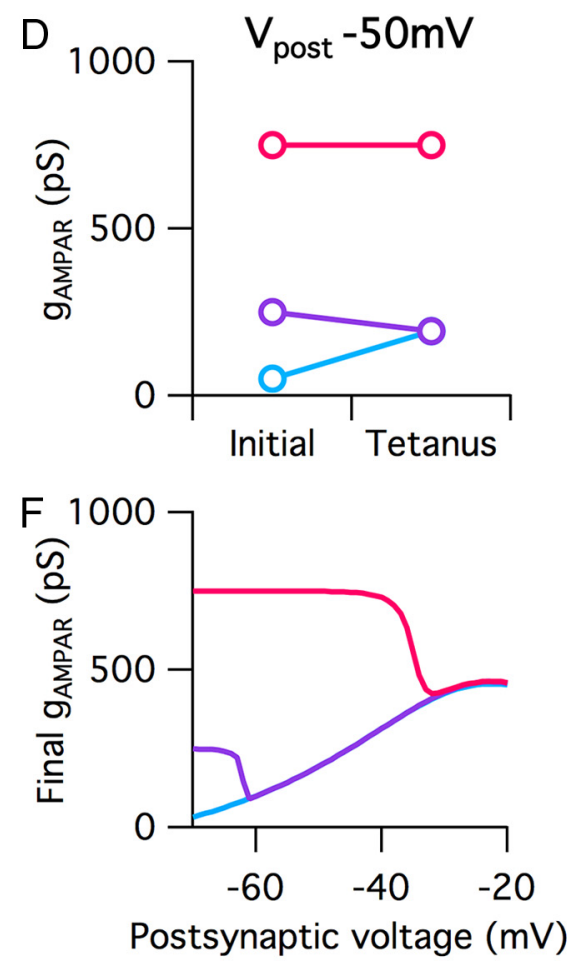

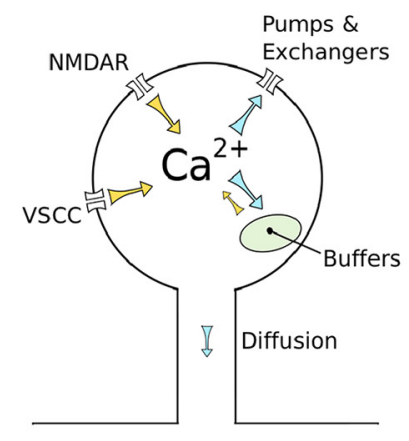

C
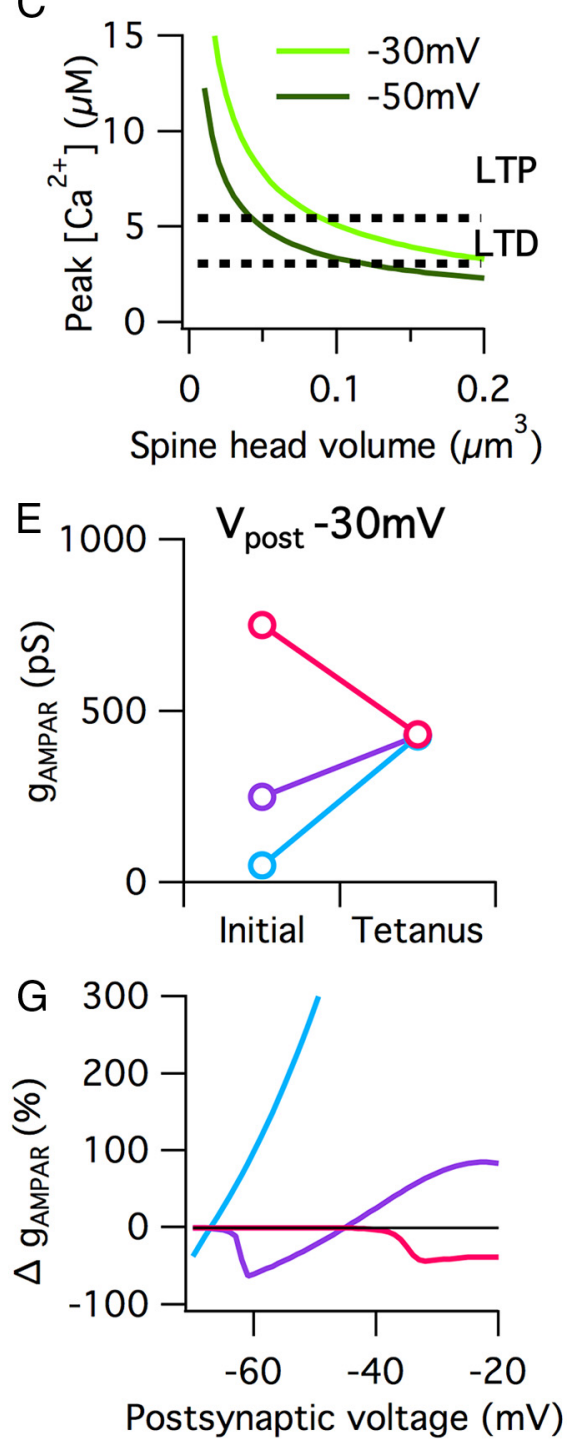

Figure 6. A biophysical spine model predicts that CA1 pyramidal synapses undercompensate. $\boldsymbol{A}$, The spine model includes electrical dynamics (left) and $\mathrm{Ca}^{2+}$ dynamics (right). $\boldsymbol{B}$, The spine's GluA and GluN conductances as a function of spine head volume. Colored symbols indicate spine volumes chosen for simulations presented in $\mathbf{D}-\boldsymbol{F}$. C, The peak spine head $\mathrm{Ca}^{2+}$ concentration obtained during burst stimulation is plotted as a function of spine head volume. Postsynaptic holding potentials of $-30 \mathrm{and}-50 \mathrm{mV}$ are in light and dark green, respectively. $\boldsymbol{D}, \boldsymbol{E}$, GluA conductance of three synapses of different initial strength following a tetanic stimulus for postsynaptic potentials of $-50 \mathrm{mV}(\boldsymbol{D})$ and $-30 \mathrm{mV}(\boldsymbol{E})$. The small, medium, and large synapses had initial spine head volumes of $0.01,0.05$, and $0.15 \mu \mathrm{m}^{3}$, respectively. $\boldsymbol{F}$, Final synaptic strength (GluA conductance) for the small, medium, and large synapses following a tetanic plasticity-inducing stimulus for a range of postsynaptic potentials. $\mathbf{G}$, Relative change in synaptic strength for same data as $\boldsymbol{F}$.

Most CA1 pyramidal dendritic spine heads have volumes of $\sim 0.01-0.06 \mu \mathrm{m}^{3}$, but the largest can have volumes of at least $\sim 0.3 \mu \mathrm{m}^{3}$ (Harris and Stevens, 1989; Mishchenko et al., 2010). We select three spine sizes spanning this distribution: 0.01, 0.05, and $0.15 \mu \mathrm{m}^{3}$ (Fig. $6 B$ ). We subject the synapses on these spines to a typical experimental protocol used to induce long-term potentiation and spine head enlargement: presynaptic stimulation (100 pulses at 100 $\mathrm{Hz}$ ) coupled with postsynaptic depolarization (Kauer et al., 1988; Cummings et al., 1996; Ngezahayo et al., 2000; Harvey and Svoboda, 2007). Varying the postsynaptic potential regulates the amount of $\mathrm{Mg}^{+}$ block of the synaptic GluNs (Jahr and Stevens, 1990), and therefore the amount of $\mathrm{Ca}^{2+}$ influx to the spine. Synaptic plasticity is triggered by the $\mathrm{Ca}^{2+}$ influx during the induction protocol (Fig. 6D,E).

At a moderate holding potential of $-50 \mathrm{mV}$, the small synapse potentiates, the medium synapse depresses, and the large synapse does not change (Fig. 6D). Importantly, the small and medium synapses converge to the same final strength, as expected for an undercompensating synapse (Fig. 2Aii). The $\mathrm{Ca}^{2+}$ concentration in the large spine does not reach the threshold for LTD, making it resistant to this induction protocol.

Upon repeating the experiment at a more depolarized postsynaptic holding potential of $-30 \mathrm{mV}$, all three synapses converge to the same strength (Fig. $6 E$ ). The final stable synaptic strength is greater than for the $-50 \mathrm{mV}$ holding potential, as predicted for undercompensating synapses (Fig. 2Cii). The large synapse's depression demonstrates how strong persistent synapses could still be reset under this scheme when given a suitably strong stimulus. We repeat the simulations for a large range of postsynaptic holding potentials (Fig. $6 F, G$ ). The weakest synapse is plastic over the entire stimulus range, while the strongest synapse is mostly resistant to change. Notably, an identical stimulus can result in different plasticity outcomes depending on the initial synaptic strength (Fig. 6G). Our model therefore suggests that CA1 pyramidal neuron spines undercompensate.

Because the simulation results we present are for only one set of parameters, we also test cases where GluN number, GluA number, $\mathrm{CaV}$ number, buffer concentration, buffer off rate, pump efficacy, spine neck diameter, and spine neck length are both doubled and halved. For all parameter variations, the model retains the qualitative features of undercompensation (data not shown). 
Spine volume can influence nanodomain $\mathrm{Ca}^{2+}$ signaling

In the models used for simulations above, we assume that the volume-averaged $\mathrm{Ca}^{2+}$ concentration in the spine is the signal read by the $\mathrm{Ca}^{2+}$-sensing molecules. This does not account for the possible roles of local $\mathrm{Ca}^{2+}$ signaling either near the pore of single channels (nanodomains) or near small clusters of channels (microdomains) (Augustine et al., 2003). Evidence supporting this assumption includes findings that EGTA, a $\mathrm{Ca}^{2+}$ chelator that binds too slowly to affect nanodomains, blocks hippocampal LTP (Lynch et al., 1983) and perirhinal LTD (Cho et al., 2001). EGTA and BAPTA are also equally effective at blocking neocortical LTD (Egger et al., 1999), spike timing-dependent plasticity (STDP) (Nevian and Sakmann, 2006), and GluN-dependent CaMKII activation (Lee et al., 2009). Finally, postsynaptic $\mathrm{Ca}^{2+}$ uncaging, predicted to be more dilute and diffuse than physiological $\mathrm{Ca}^{2+}$ nanodomains, is sufficient to induce both LTP and LTD (Yang et al., 1999).

Nevertheless, there are also data indicating a role for local $\mathrm{Ca}^{2+}$ interactions in some forms of synaptic plasticity (Hoffman et al., 2002; Yasuda et al., 2003; Lee et al., 2009). This possibility might pose a significant problem for the theory we propose because, if synaptic plasticity were found to be dependent on nanodomain $\mathrm{Ca}^{2+}$ signaling, then spine size changes might not influence the process. Hence, it is important to understand whether the bulk spine $\left[\mathrm{Ca}^{2+}\right]$, which is determined by spine volume, can affect $\mathrm{Ca}^{2+}$ concentration changes near the pore of individual CaVs.

To address this issue, we use a previously published molecular-level model of a hippocampal CA1-pyramidal neuron dendritic spine (Fig. 7A) adapted from Keller et al. (2008). Nanodomain signaling was tested as follows. Initially, we elevate $\left[\mathrm{Ca}^{2+}\right]$ throughout the bulk of the spine by opening multiple GluNs. Then, a single $\mathrm{Ca}_{\mathrm{v}} 1$ (L-type) channel is allowed to open and close stochastically (with an open probability of 0.1 ) in addition to the continually open GluNs (Fig. $7 B$ ). We choose the L-type $\mathrm{CaV}$ because it is the only $\mathrm{CaV}$ that has been implicated in local postsynaptic $\mathrm{Ca}^{2+}$ signaling involved in synaptic plasticity (Yasuda et al., 2003; Lee et al., 2009). When the $\mathrm{CaV}$ is open, it influxes $\mathrm{Ca}^{2+}$ ions at a rate of $3.3 \times 10^{6} \mathrm{~s}^{-1}$, rapidly causing a localized elevation of $\mathrm{Ca}^{2+}$ concentration near the channel pore, where $\mathrm{Ca}^{2+}$ buffers are not in their equilibrium binding state (Fig. 7C). We repeat the stochastic simulation 10 times and measure the mean $\mathrm{Ca}^{2+}$ concentration while the channel is open as a function of distance from the channel for a range of spine volumes (Fig. $7 D, E$ ). Decreasing the spine volume inare \pm SD.
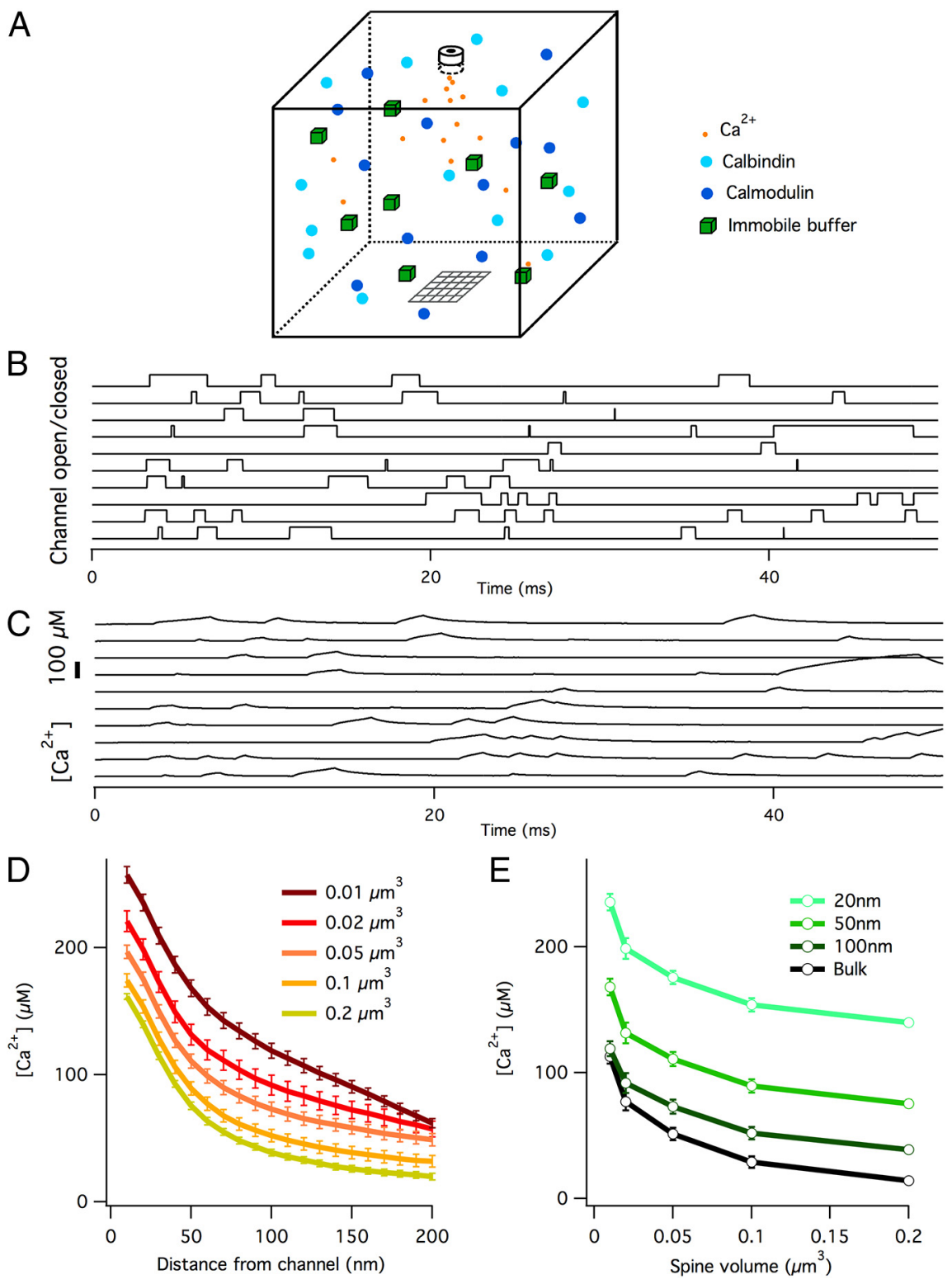

Figure 7. Spine size influences $\mathrm{Ca}^{2+}$ nanodomain signaling in a molecular model of a $\mathrm{CA} 1$ pyramidal neuron dendritic spine. $A$, Schematic diagram of molecular spine model (MCell simulator). Shown are $\mathrm{Ca}^{2+}$ ions, calbindin, calmodulin, and a fast immobile endogenous buffer. L-type $\mathrm{Ca}^{2+}$ channel at top of spine, $\mathrm{Ca}^{2+}$ transparent patch (gray mesh) at bottom allowing $\mathrm{Ca}^{2+}$ escape to spine neck and dendrite. Not shown are $20 \mathrm{GluNs}$ (distributed randomly across top surface of spine), PCMA Ca ${ }^{2+}$ pumps, NCXs, and leak $\mathrm{Ca}^{2+}$ influx channels (distributed randomly across entire surface of spine). B, State of the L-type $\mathrm{Ca}^{2+}$ channel (open or closed) as a function of time for 10 different trials. Note that the channel open and closes stochastically. C, Mean $\mathrm{Ca}^{2+}$ concentration throughout the spine as a function of time for the same 10 trials depicted in $\boldsymbol{B}$. When the $\mathrm{L}$-type $\mathrm{Ca}^{2+}$ channel opens, $\left[\mathrm{Ca}^{2+}\right.$ ] is rapidly elevated. $\boldsymbol{D}, \mathrm{Local} \mathrm{Ca}^{2+}$ concentration as a function of distance from an open $\mathrm{L}$-type $\mathrm{Ca}^{2+}$ channel. Each curve represents a different spine volume. Error bars are $\pm S D$. $E$, Local $\mathrm{Ca}^{2+}$ concentration as a function of spine volume at various distances from an open $\mathrm{L}$-type $\mathrm{Ca}^{2+}$ channel. Same data as in $\boldsymbol{D}$. Bulk, indicates mean $\mathrm{Ca}^{2+}$ concentration over the entire spine volume. Error bars

creased the $\mathrm{Ca}^{2+}$ concentration at all distances from the channel, because the bulk $\mathrm{Ca}^{2+}$ concentration was large enough to substantially add to the local $\mathrm{Ca}^{2+}$ concentration. The effect was greatest for small spine volumes $<0.05 \mu \mathrm{m}^{3}$. Therefore, nanodomain signaling is sensitive to changes in spine volume at hippocampal synapses.

In addition to the mechanism described above, there are two further mechanisms through which spine size could regulate local $\mathrm{Ca}^{2+}$ signaling events. First, because small spines have a greater surface-to-volume ratio than large spines, any mobile 
$\mathrm{Ca}^{2+}$-sensing molecules are statistically more likely to diffuse closer to membrane-bound $\mathrm{Ca}^{2+}$ sources in smaller spines than in larger spines. This mechanism likely exists at dendritic spines in all brain regions. Second, the bulk $\mathrm{Ca}^{2+}$ concentration sets the level of endogenous buffer saturation. Hence, if bulk $\mathrm{Ca}^{2+}$ concentrations are sufficiently elevated, there is less free buffer available to restrict the $\mathrm{Ca}^{2+}$ influxing through the channel pore, so elevating microdomain $\mathrm{Ca}^{2+}$ signals - a mechanism originally proposed to explain activity-dependent presynaptic facilitation (Neher, 1998). Because this mechanism exists only in situations where buffers are relatively close to saturation, it had only negligible effect in our simulations of hippocampal spines (data not shown). Future experimental data may clarify whether this mechanism exists at dendritic spines in other brain regions. Importantly, both of these additional mechanisms are consistent with the prediction from our earlier simulations, that hippocampal spines undercompensate (Fig. 6).

In summary, our simulation results (Fig. 7) support the viewpoint that even if synaptic plasticity relies on nanodomain or microdomain $\mathrm{Ca}^{2+}$ signaling, spine volume will still implement undercompensation as smaller spines experience larger nanodomain $\mathrm{Ca}^{2+}$ signals than large spines.

\section{Discussion}

Our results introduce several new and general conclusions. First, we provide a framework for understanding the functional consequences of spine structural plasticity by delineating the three scenarios of $\mathrm{Ca}^{2+}$ overcompensation, undercompensation, or exact compensation (Fig. 1). Second, we demonstrate the consequences of these three scenarios for the form of synaptic information storage (Fig. 2), the long-term retention of synaptic strength (Figs. 3, 4), the robustness of memory storage to intrinsic synaptic strength fluctuations (Fig. 4), and the distributions of synaptic strength (Fig. 5).

Biophysical simulation of a hippocampal CA1 pyramidal neuron spine suggests that synapses onto these neurons likely undercompensate (Fig. 6). This prediction unifies several disparate pieces of evidence: (1) GluN immunoreactivity (Takumi et al., 1999; Racca et al., 2000; Ganeshina et al., 2004) and GluN EPSC amplitude (Nimchinsky et al., 2004; Noguchi et al., 2005; Sobczyk et al., 2005) are not correlated with spine size; (2) focal glutamate uncaging causes larger $\mathrm{Ca}^{2+}$ fluorescence transients in small spines than in large spines (Nimchinsky et al., 2004; Noguchi et al., 2005; Sobczyk et al., 2005); (3) synapses on small spines are easier to potentiate than synapses on large spines (Matsuzaki et al., 2004); (4) weak synapses potentiate more than strong synapses (Larkman et al., 1992; Bi and Poo, 1998; Debanne et al., 1999); (5) potentiated synapses become "locked in" at high strength (O'Connor et al., 2005); (6) repeated LTP saturates (McNaughton et al., 1978; Dudek and Bear, 1993; O'Connor et al., 2005); (7) spine size and synaptic strength distributions are unimodal with a nonzero peak (Harris and Stevens, 1989; Sayer et al., 1990; Schikorski and Stevens, 1997; O'Brien et al., 1998; Yasumatsu et al., 2008; Enoki et al., 2009; Minerbi et al., 2009; Mishchenko et al., 2010; Loewenstein et al., 2011); and (8) longitudinal studies in hippocampal cultures find a negative correlation between momentary synaptic strength and the subsequent change in synaptic strength (Yasumatsu et al., 2008; Minerbi et al., 2009; Loewenstein et al., 2011). Nevertheless, the prediction of our model is strongly dependent on the assumed relationship between spine head volume and GluN number. Future data on the relationship between spine size and GluN number, CaVs, extrusion mechanisms, and endogenous buffers can be used to refine the model to make more quantitative predictions.

There is no known mechanistic reason why spine structural plasticity should necessarily be tethered to synaptic plasticity. The two processes are mediated by signaling pathways that are at least partly independent from one another (Cingolani and Goda, 2008), suggesting that they are coregulated for functional reasons. Our results suggest that the spine size-to-synaptic efficacy relationship is maintained to imprint a strength dependence on the synaptic plasticity rule that preferentially stabilizes some synaptic strengths. Although this mechanism helps to prevent runaway synaptic plasticity, it is not homeostatic. Homeostatic plasticity typically entails a feedback mechanism that returns neural activity to a set point (Davis, 2006), while here there is no such feedback.

What is the relevance of different $\mathrm{Ca}^{2+}$-permeable channel types for the framework we describe here? In our biophysical spine model (Fig. 6), we include one generic GluN type and four different $\mathrm{CaV}$ types. Different GluNs and CaVs can have different neurotransmitter affinities, voltage dependences, and time courses of activation/inactivation. This implies that each channel type will respond differently to a given presynaptic and postsynaptic activity pattern. How this feature impacts our scenarios of undercompensation, overcompensation, or exact compensation will depend on how the number of channels of each individual type scales with spine size. For example, consider a hypothetical case where $\mathrm{Ca}_{\mathrm{v}} 3$ (T-type) number is proportional to spine volume, but $\mathrm{Ca}_{\mathrm{v}} 1$ (L-type) number is fixed and independent of spine volume. Because $\mathrm{Ca}_{v} 3$ s are activated at relatively modest potentials, whereas $\mathrm{Ca}_{\mathrm{v}} 1 \mathrm{~s}$ are activated only at very depolarized potentials (Magee and Johnston, 1995), this configuration would make $\mathrm{Ca}^{2+}$ influx behave as if in the compensating scenario for stimuli that result in modest depolarizations, but in the undercompensating scenario for stimuli that induced large depolarizations. Hence, a single spine might simultaneously belong to multiple scenarios, depending on the type of stimuli it is subject to.

The plasticity induction protocols we employ in this study were designed to mimic classic LTP and LTD induction protocols where repeated presynaptic stimulation is accompanied by postsynaptic depolarization (Artola et al., 1990; Dudek and Bear, 1992; Mayford et al., 1995; Ngezahayo et al., 2000; Cho et al., 2001; Ismailov et al., 2004; O'Connor et al., 2005). An interesting future extension of our model would be to explore the effects of undercompensation and overcompensation for STDP protocols, where fine time-scale differences between presynaptic and postsynaptic events determine the direction of plasticity (Caporale and Dan, 2008). However, at present no theoretical model of $\mathrm{Ca}^{2+}$-dependent synaptic plasticity can fully account for the data from STDP experiments (for a discussion, see Shouval et al., 2010). Should future experiments resolve this issue, the general framework of exact, under-, and overcompensation we introduce here could be readily applied to the problem.

The consequences of certain weight-dependent synaptic plasticity rules for long-term synaptic strength dynamics have been considered previously (van Rossum et al., 2000; Rubin et al., 2001; Gütig et al., 2003; Zou and Destexhe, 2007; Billings and van Rossum, 2009). However, these phenomenological models often predict that strong synapses are the least stable (but see Shouval, 2005), whereas experimental data suggest that strong synapses and large spines are the most stable (Grutzendler et al., 2002; Trachtenberg et al., 2002; Holtmaat et al., 2005; Zuo et al., 2005; Knott et al., 2006). This stability is successfully explained by the 
mechanisms from spine structural plasticity that we propose here.

A second class of models of synaptic strength stability relies on intricate molecular cascades with multiple stable states (Lisman and Zhabotinsky, 2001; Hayer and Bhalla, 2005; Graupner and Brunel, 2007). However, the model we propose has an advantage over these previous models. Although spines in vivo can exist stably for many months (Grutzendler et al., 2002; Trachtenberg et al., 2002; Holtmaat et al., 2005; Zuo et al., 2005; Knott et al., 2006), the spine and PSD are tiny devices (volume $<1 \mathrm{fl}$ ), implying that the molecular reactions at the synapse involve a small number of particles and may therefore be noisy (Franks and Sejnowski, 2002; Keller et al., 2008). When LTP cascades are modeled stochastically, spontaneous transitions are found to occur between states that are stable in an equivalent deterministic model (Bhalla, 2004). Changing parameters to reduce the spontaneous transitions also makes the system insensitive to stimuli (but see Miller et al., 2005). In contrast, undercompensating and overcompensating synapses can both override the effects of noise on stability (Fig. 4).

The framework we propose makes specific experimental predictions. Undercompensating synapses should show the following properties collectively: (1) stable strong synapses but plastic weak synapses; (2) synaptic retention time should increase dramatically with synaptic strength; (3) a plasticity-inducing stimulus should drive all stimulated synapses toward a single common strength; (4) the stable synaptic strength should be an increasing, continuous function of stimulus strength; (5) the stable strength can be varied by enhancing or reducing $\mathrm{Ca}^{2+}$ influx to the spine; and (6) the distribution of synaptic strengths should be unimodal with a central nonzero peak. Overcompensating synapses, in contrast, should show collectively the following: (1) individual synapses should be most stable at a maximum or minimum strength, but not at intermediate strengths; (2) a plasticity-inducing stimulus should potentiate all synapses with a strength greater than a certain threshold and depress all synapses with a strength weaker than the same threshold; (3) the threshold should be a continuously increasing function of stimulus strength; (4) the threshold can be varied by enhancing or reducing $\mathrm{Ca}^{2+}$ influx to the spine; (5) there should be some additional mechanism to limit synaptic strength at its maximum and minimum values; and (6) synaptic strength distributions should appear bimodal.

A powerful approach to test the predictions of our model will be by pharmacological or genetic dissociation of spine size from synaptic strength (Zhou et al., 2004; Wang et al., 2007). For example, manipulations that permit synaptic plasticity while blocking spine structural plasticity would make synapses behave as if in the compensating mode where our model predicts the following: (1) individual synaptic strengths drift at an elevated rate that is independent of synaptic strength; (2) potentiated synapses rapidly decay to naive strengths; and (3) synaptic strength distributions spread. A further critical experiment would be the tracking of individual synaptic strengths over time in vivo. Correlating spine and synaptic strength changes with physiological activity patterns could uncover strength-dependent plasticity rules.

In summary, our findings provide a general theoretical framework for understanding how dendritic spine structural plasticity actively regulates synaptic learning rules to stabilize some synaptic strengths over others. This mechanism allows synapses to retain both the features of rapid plasticity and of persistent information storage without precise tuning of the molecular synaptic plasticity cascade.

\section{References}

Arellano JI, Benavides-Piccione R, Defelipe J, Yuste R (2007) Ultrastructure of dendritic spines: correlation between synaptic and spine morphologies. Front Neurosci 1:131-143.

Artola A, Bröcher S, Singer W (1990) Different voltage-dependent thresholds for inducing long-term depression and long-term potentiation in slices of rat visual cortex. Nature 347:69-72.

Augustine GJ, Santamaria F, Tanaka K (2003) Local calcium signaling in neurons. Neuron 40:331-346.

Barbour B, Brunel N, Hakim V, Nadal JP (2007) What can we learn from synaptic weight distributions? Trends Neurosci 30:622-629.

Barrett AB, van Rossum MC (2008) Optimal learning rules for discrete synapses. PLoS Comput Biol 4:e1000230.

Bear MF, Cooper LN, Ebner FF (1987) A physiological basis for a theory of synapse modification. Science 237:42-48.

Bhalla US (2004) Signaling in small subcellular volumes. I. Stochastic and diffusion effects on individual pathways. Biophys J 87:733-744.

Bi GQ, Poo MM (1998) Synaptic modifications in cultured hippocampal neurons: dependence on spike timing, synaptic strength, and postsynaptic cell type. J Neurosci 18:10464-10472.

Bienenstock EL, Cooper LN, Munro PW (1982) Theory for the development of neuron selectivity: orientation specificity and binocular interaction in visual cortex. J Neurosci 2:32-48.

Billings G, van Rossum MC (2009) Memory retention and spike-timingdependent plasticity. J Neurophysiol 101:2775-2788.

Blanpied TA, Kerr JM, Ehlers MD (2008) Structural plasticity with preserved topology in the postsynaptic protein network. Proc Natl Acad Sci U S A 105:12587-12592.

Bloodgood BL, Sabatini BL (2005) Neuronal activity regulates diffusion across the neck of dendritic spines. Science 310:866-869.

Bloodgood BL, Sabatini BL (2007) Nonlinear regulation of unitary synaptic signals by $\mathrm{CaV}(2.3)$ voltage-sensitive calcium channels located in dendritic spines. Neuron 53:249-260.

Caporale N, Dan Y (2008) Spike timing-dependent plasticity: a Hebbian learning rule. Annu Rev Neurosci 31:25-46.

Carlin KP, Jiang Z, Brownstone RM (2000) Characterization of calcium currents in functionally mature mouse spinal motoneurons. Eur J Neurosci 12:1624-1634.

Catterall WA, Perez-Reyes E, Snutch TP, Striessnig J (2005) International Union of Pharmacology. XLVIII. Nomenclature and structure-function relationships of voltage-gated calcium channels. Pharmacol Rev 57:411-425.

Cho K, Aggleton JP, Brown MW, Bashir ZI (2001) An experimental test of the role of postsynaptic calcium levels in determining synaptic strength using perirhinal cortex of rat. J Physiol 532:459-466.

Cingolani LA, Goda Y (2008) Actin in action: the interplay between the actin cytoskeleton and synaptic efficacy. Nat Rev Neurosci 9:344-356.

Collingridge GL, Olsen RW, Peters J, Spedding M (2009) A nomenclature for ligand-gated ion channels. Neuropharmacology 56:2-5.

Cormier RJ, Greenwood AC, Connor JA (2001) Bidirectional synaptic plasticity correlated with the magnitude of dendritic calcium transients above a threshold. J Neurophysiol 85:399-406.

Cummings JA, Mulkey RM, Nicoll RA, Malenka RC (1996) Ca2 + signaling requirements for long-term depression in the hippocampus. Neuron 16:825-833.

Davis GW (2006) Homeostatic control of neural activity: from phenomenology to molecular design. Annu Rev Neurosci 29:307-323.

Debanne D, Gähwiler BH, Thompson SM (1999) Heterogeneity of synaptic plasticity at unitary CA3-CA1 and CA3-CA3 connections in rat hippocampal slice cultures. J Neurosci 19:10664-10671.

Dudek SM, Bear MF (1992) Homosynaptic long-term depression in area CA1 of hippocampus and effects of N-methyl-D-aspartate receptor blockade. Proc Natl Acad Sci U S A 89:4363-4367.

Dudek SM, Bear MF (1993) Bidirectional long-term modification of synaptic effectiveness in the adult and immature hippocampus. J Neurosci 13:2910-2918.

Egger V, Feldmeyer D, Sakmann B (1999) Coincidence detection and changes of synaptic efficacy in spiny stellate neurons in rat barrel cortex. Nat Neurosci 2:1098-1105

Enoki R, Hu YL, Hamilton D, Fine A (2009) Expression of long-term plasticity at individual synapses in hippocampus is graded, bidirectional, and mainly presynaptic: optical quantal analysis. Neuron 62:242-253. 
Franks KM, Sejnowski TJ (2002) Complexity of calcium signaling in synaptic spines. Bioessays 24:1130-1144.

Fusi S, Abbott LF (2007) Limits on the memory storage capacity of bounded synapses. Nat Neurosci 10:485-493.

Fusi S, Senn W (2006) Eluding oblivion with smart stochastic selection of synaptic updates. Chaos 16:026112.

Ganeshina O, Berry RW, Petralia RS, Nicholson DA, Geinisman Y (2004) Differences in the expression of AMPA and NMDA receptors between axospinous perforated and nonperforated synapses are related to the configuration and size of postsynaptic densities. J Comp Neurol 468:86-95.

Gold JI, Bear MF (1994) A model of dendritic spine Ca2+ concentration exploring possible bases for a sliding synaptic modification threshold. Proc Natl Acad Sci U S A 91:3941-3945.

Graupner M, Brunel N (2007) STDP in a bistable synapse model based on CaMKII and associated signaling pathways. PLoS Comput Biol 3:e221.

Grunditz A, Holbro N, Tian L, Zuo Y, Oertner TG (2008) Spine neck plasticity controls postsynaptic calcium signals through electrical compartmentalization. J Neurosci 28:13457-13466

Grutzendler J, Kasthuri N, Gan WB (2002) Long-term dendritic spine stability in the adult cortex. Nature 420:812-816.

Gütig R, Aharonov R, Rotter S, Sompolinsky H (2003) Learning input correlations through nonlinear temporally asymmetric Hebbian plasticity. J Neurosci 23:3697-3714.

Hansel C, Artola A, Singer W (1997) Relation between dendritic Ca2 + levels and the polarity of synaptic long-term modifications in rat visual cortex neurons. Eur J Neurosci 9:2309-2322.

Harris KM, Stevens JK (1989) Dendritic spines of CA 1 pyramidal cells in the rat hippocampus: serial electron microscopy with reference to their biophysical characteristics. J Neurosci 9:2982-2997.

Harris KM, Jensen FE, Tsao B (1992) Three-dimensional structure of dendritic spines and synapses in rat hippocampus (CA1) at postnatal day 15 and adult ages: implications for the maturation of synaptic physiology and long-term potentiation. J Neurosci 12:2685-2705.

Harvey CD, Svoboda K (2007) Locally dynamic synaptic learning rules in pyramidal neuron dendrites. Nature 450:1195-1200.

Hayer A, Bhalla US (2005) Molecular switches at the synapse emerge from receptor and kinase traffic. PLoS Comput Biol 1:137-154.

Hoffman DA, Sprengel R, Sakmann B (2002) Molecular dissection of hippocampal theta-burst pairing potentiation. Proc Natl Acad Sci U S A 99:7740-7745.

Holtmaat AJ, Trachtenberg JT, Wilbrecht L, Shepherd GM, Zhang X, Knott GW, Svoboda K (2005) Transient and persistent dendritic spines in the neocortex in vivo. Neuron 45:279-291.

Huang SJ, Robinson DW (1998) Activation and inactivation properties of voltage-gated calcium currents in developing cat retinal ganglion cells. Neuroscience 85:239-247.

Ismailov I, Kalikulov D, Inoue T, Friedlander MJ (2004) The kinetic profile of intracellular calcium predicts long-term potentiation and long-term depression. J Neurosci 24:9847-9861.

Jahr CE, Stevens CF (1990) Voltage dependence of NMDA-activated macroscopic conductances predicted by single-channel kinetics. J Neurosci 10:3178-3182.

Kalantzis G, Shouval HZ (2009) Structural plasticity can produce metaplasticity. PLoS ONE 4:e8062.

Kauer JA, Malenka RC, Nicoll RA (1988) A persistent postsynaptic modification mediates long-term potentiation in the hippocampus. Neuron 1:911-917.

Keller DX, Franks KM, Bartol TM Jr, Sejnowski TJ (2008) Calmodulin activation by calcium transients in the postsynaptic density of dendritic spines. PLoS One 3:e2045.

Knott GW, Holtmaat A, Wilbrecht L, Welker E, Svoboda K (2006) Spine growth precedes synapse formation in the adult neocortex in vivo. Nat Neurosci 9:1117-1124.

Larkman A, Hannay T, Stratford K, Jack J (1992) Presynaptic release probability influences the locus of long-term potentiation. Nature 360:70-73.

Lee SJ, Escobedo-Lozoya Y, Szatmari EM, Yasuda R (2009) Activation of CaMKII in single dendritic spines during long-term potentiation. Nature 458:299-304.

Lisman J (1989) A mechanism for the Hebb and the anti-Hebb processes underlying learning and memory. Proc Natl Acad Sci U S A 86: 9574-9578.

Lisman JE, Zhabotinsky AM (2001) A model of synaptic memory: a CaM-
KII/PP1 switch that potentiates transmission by organizing an AMPA receptor anchoring assembly. Neuron 31:191-201.

Loewenstein Y, Kuras A, Rumpel S (2011) Multiplicative dynamics underlie the emergence of the log-normal distribution of spine sizes in the neocortex in vivo. J Neurosci 31:9481-9488.

Lynch G, Larson J, Kelso S, Barrionuevo G, Schottler F (1983) Intracellular injections of EGTA block induction of hippocampal long-term potentiation. Nature 305:719-721.

Magee JC, Johnston D (1995) Characterization of single voltage-gated $\mathrm{Na}+$ and $\mathrm{Ca} 2+$ channels in apical dendrites of rat CA1 pyramidal neurons. J Physiol 487:67-90.

Malenka RC, Bear MF (2004) LTP and LTD: an embarrassment of riches. Neuron 44:5-21.

Matsuzaki M, Ellis-Davies GC, Nemoto T, Miyashita Y, Iino M, Kasai H (2001) Dendritic spine geometry is critical for AMPA receptor expression in hippocampal CA1 pyramidal neurons. Nat Neurosci 4:1086-1092.

Matsuzaki M, Honkura N, Ellis-Davies GC, Kasai H (2004) Structural basis of long-term potentiation in single dendritic spines. Nature 429:761-766.

Mayford M, Wang J, Kandel ER, O’Dell TJ (1995) CaMKII regulates the frequency-response function of hippocampal synapses for the production of both LTD and LTP. Cell 81:891-904.

McNaughton BL, Douglas RM, Goddard GV (1978) Synaptic enhancement in fascia dentata: cooperativity among coactive afferents. Brain Res 157:277-293.

Miller P, Zhabotinsky AM, Lisman JE, Wang XJ (2005) The stability of a stochastic CaMKII switch: dependence on the number of enzyme molecules and protein turnover. PLoS Biol 3:e107.

Milner B, Squire LR, Kandel ER (1998) Cognitive neuroscience and the study of memory. Neuron 20:445-468.

Minerbi A, Kahana R, Goldfeld L, Kaufman M, Marom S, Ziv NE (2009) Long-term relationships between synaptic tenacity, synaptic remodeling, and network activity. PLoS Biol 7:e1000136.

Mishchenko Y, Hu T, Spacek J, Mendenhall J, Harris KM, Chklovskii DB (2010) Ultrastructural analysis of hippocampal neuropil from the connectomics perspective. Neuron 67:1009-1020.

Morris RG, Moser EI, Riedel G, Martin SJ, Sandin J, Day M, O'Carroll C (2003) Elements of a neurobiological theory of the hippocampus: the role of activity-dependent synaptic plasticity in memory. Philos Trans R Soc Lond, B, Biol Sci 358:773-786.

Mysore SP, Tai CY, Schuman EM (2007) Effects of N-cadherin disruption on spine morphological dynamics. Front Cell Neurosci 1:1.

Neher E (1998) Usefulness and limitations of linear approximations to the understanding of $\mathrm{Ca}++$ signals. Cell Calcium 24:345-357.

Nevian T, Sakmann B (2006) Spine $\mathrm{Ca}^{2+}$ signaling in spike-timingdependent plasticity. J Neurosci 26:11001-11013.

Ngezahayo A, Schachner M, Artola A (2000) Synaptic activity modulates the induction of bidirectional synaptic changes in adult mouse hippocampus. J Neurosci 20:2451-2458.

Nimchinsky EA, Yasuda R, Oertner TG, Svoboda K (2004) The number of glutamate receptors opened by synaptic stimulation in single hippocampal spines. J Neurosci 24:2054-2064.

Noguchi J, Matsuzaki M, Ellis-Davies GC, Kasai H (2005) Spine-neck geometry determines NMDA receptor-dependent $\mathrm{Ca} 2+$ signaling in dendrites. Neuron 46:609-622.

Nusser Z, Lujan R, Laube G, Roberts JD, Molnar E, Somogyi P (1998) Cell type and pathway dependence of synaptic AMPA receptor number and variability in the hippocampus. Neuron 21:545-559.

O’Brien RJ, Kamboj S, Ehlers MD, Rosen KR, Fischbach GD, Huganir RL (1998) Activity-dependent modulation of synaptic AMPA receptor accumulation. Neuron 21:1067-1078.

O'Connor DH, Wittenberg GM, Wang SS (2005) Dissection of bidirectional synaptic plasticity into saturable unidirectional processes. J Neurophysiol 94:1565-1573.

Petersen CC, Malenka RC, Nicoll RA, Hopfield JJ (1998) All-or-none potentiation at CA3-CA1 synapses. Proc Natl Acad Sci U S A 95:4732-4737.

Racca C, Stephenson FA, Streit P, Roberts JD, Somogyi P (2000) NMDA receptor content of synapses in stratum radiatum of the hippocampal CA1 area. J Neurosci 20:2512-2522.

Rubin J, Lee DD, Sompolinsky H (2001) Equilibrium properties of temporally asymmetric Hebbian plasticity. Phys Rev Lett 86:364-367. 
Sabatini BL, Oertner TG, Svoboda K (2002) The life cycle of $\mathrm{Ca}(2+)$ ions in dendritic spines. Neuron 33:439-452.

Sayer RJ, Friedlander MJ, Redman SJ (1990) The time course and amplitude of EPSPs evoked at synapses between pairs of CA3/CA1 neurons in the hippocampal slice. J Neurosci 10:826-836.

Schikorski T, Stevens CF (1997) Quantitative ultrastructural analysis of hippocampal excitatory synapses. J Neurosci 17:5858-5867.

Segev I, Koch C eds. (1998) Methods in neuronal modeling (Segev I, Koch C, eds). Cambridge, MA: MIT.

Shouval HZ (2005) Clusters of interacting receptors can stabilize synaptic efficacies. Proc Natl Acad Sci U S A 102:14440-14445.

Shouval HZ, Bear MF, Cooper LN (2002) A unified model of NMDA receptor-dependent bidirectional synaptic plasticity. Proc Natl Acad Sci U S A 99:10831-10836.

Shouval HZ, Wang SS-H, Wittenberg GM (2010) Spike timing dependent plasticity: a consequence of more fundamental learning rules. Front Comput Neurosci 4:19.

Sobczyk A, Scheuss V, Svoboda K (2005) NMDA receptor subunitdependent $\left[\mathrm{Ca}^{2+}\right]$ signaling in individual hippocampal dendritic spines. J Neurosci 25:6037-6046.

Stiles JR, Van Helden D, Bartol TM Jr, Salpeter EE, Salpeter MM (1996) Miniature endplate current rise times less than 100 microseconds from improved dual recordings can be modeled with passive acetylcholine diffusion from a synaptic vesicle. Proc Natl Acad Sci USA 93:5747-5752.

Takumi Y, Ramírez-León V, Laake P, Rinvik E, Ottersen OP (1999) Different modes of expression of AMPA and NMDA receptors in hippocampal synapses. Nat Neurosci 2:618-624.

Traboulsie A, Chemin J, Chevalier M, Quignard JF, Nargeot J, Lory P (2007)
Subunit-specific modulation of T-type calcium channels by zinc. J Physiol 578:159-171.

Trachtenberg JT, Chen BE, Knott GW, Feng G, Sanes JR, Welker E, Svoboda K (2002) Long-term in vivo imaging of experience-dependent synaptic plasticity in adult cortex. Nature 420:788-794.

van Kampen NG (1992) Stochastic processes in physics and chemistry, Ed2. Amsterdam: North-Holland.

van Rossum MC, Bi GQ, Turrigiano GG (2000) Stable Hebbian learning from spike timing-dependent plasticity. J Neurosci 20:8812-8821.

Wang XB, Yang Y, Zhou Q (2007) Independent expression of synaptic and morphological plasticity associated with long-term depression. J Neurosci 27:12419-12429.

Yang SN, Tang YG, Zucker RS (1999) Selective induction of LTP and LTD by postsynaptic [Ca2+]i elevation. J Neurophysiol 81:781-787.

Yasuda R, Sabatini BL, Svoboda K (2003) Plasticity of calcium channels in dendritic spines. Nat Neurosci 6:948-955.

Yasumatsu N, Matsuzaki M, Miyazaki T, Noguchi J, Kasai H (2008) Principles of long-term dynamics of dendritic spines. J Neurosci 28:13592-13608.

Zhou Q, Homma KJ, Poo MM (2004) Shrinkage of dendritic spines associated with long-term depression of hippocampal synapses. Neuron 44:749-757.

Zou Q, Destexhe A (2007) Kinetic models of spike-timing dependent plasticity and their functional consequences in detecting correlations. Biol Cybern 97:81-97.

Zucker RS (1999) Calcium- and activity-dependent synaptic plasticity. Curr Opin Neurobiol 9:305-313.

Zuo Y, Lin A, Chang P, Gan WB (2005) Development of long-term dendritic spine stability in diverse regions of cerebral cortex. Neuron 46:181189. 